\title{
Complexity of the Classical Kernel Functions of Potential Theory
}

\author{
Steven R. BeLL
}

\begin{abstract}
We show that the Bergman, Szegö, and Poisson kernels associated to an $n$-connected domain in the plane are not genuine functions of two complex variables. Rather, they are all given by elementary rational combinations of $n+1$ holomorphic functions of one complex variable and their conjugates. Moreover, all three kernel functions are composed of the same basic $n+1$ functions. Our results can be interpreted as saying that the kernel functions are simpler than one might expect. We also prove, however, that the kernels cannot be too simple by showing that the only finitely connected domains in the plane whose Bergman or Poisson kernels are rational functions are the simply connected domains which can be mapped onto the unit disc by a rational biholomorphic mapping. This leads to a proof that the classical Green's function associated to a finitely connected domain in the plane is one half the logarithm of a real valued rational function if and only if the domain is simply connected and there is a rational biholomorphic map of the domain onto the unit disc. We also characterize those domains in the plane that have rational Szegő kernel functions.
\end{abstract}

1. Introduction. The Bergman and Szegő kernels associated to a bounded domain in the plane carry encoded within them an astonishing amount of information about the domain. Conformal mappings onto canonical regions, classical domain functions, and other important objects of potential theory can be expressed simply in terms of the Bergman and Szegö kernels. It is therefore tempting to believe that these kernels are extremely complex and difficult to compute. The purpose of this paper is to show that the kernel functions are not nearly as complex as one might suspect, and that they are not bona fide functions of two complex variables. 
We shall study the kernel functions on a finitely connected domain in the plane such that no boundary component reduces to a point. Such a domain can be mapped biholomorphically to a domain $\Omega$ with $C^{\infty}$ smooth boundary, i.e., a domain whose boundary $b \Omega$ is given by finitely many non-intersecting $C^{\infty}$ simple closed curves. Our problem is to determine $K(z, w)$ and $S(z, w)$ at any given ordered pair of points $(z, w)$ in $(\Omega \times \Omega)$. We shall see that, once the boundary values of finitely many basic functions of one variable have been determined, the kernels become known at all points $(z, w)$. Furthermore, the basic functions which comprise the kernel functions are all solutions to explicit Kerzman-Stein integral equations. After we treat the case of domains with smooth boundaries, it will be easy to pull back our results to more general domains by means of transformation formulas for the kernel functions.

In Section 6, we give conditions on a domain for its Bergman or Szegő kernel function to be a rational function. We prove, for example, that the Bergman kernel associated to a finitely connected domain is rational exactly when the domain is simply connected and is biholomorphic to the unit disc via a rational mapping. Thus, the domains whose Bergman kernels are rational are precisely those domains whose kernels can be seen to be rational by means of an elementary application of the transformation formula for the kernel functions under biholomorphic maps and the fact that the kernel for the unit disc is rational. This result about the Bergman kernel has as a corollary that the Green's function associated to a finitely connected domain is one half the logarithm of a rational function if and only if the domain is simply connected and there is a rational biholomorphic map of the domain onto the unit disc.

In Section 7, we show how the Poisson kernel can be expressed in terms of the Szegö kernel, and thereby shed light on the degree of complexity of the Poisson kernel.

Our results are most interesting in case the domain under study is multiply connected. However, to illustrate the flavor of our results, we take a moment here to state analogues of our theorems for a bounded simply connected domain $\Omega$ with $C^{\infty}$ smooth boundary. (The assumption of $C^{\infty}$ smooth boundary can be greatly reduced, but since we just want to motivate our results, we shall not consider these technical points here.) Let $a$ be a fixed point in $\Omega$ and let $f_{a}(z)$ denote the Riemann mapping function mapping $\Omega$ one-to-one onto the unit disc $D_{1}(0)$ with $f_{a}(a)=0$ and $f_{a}^{\prime}(a)>0$. This Riemann map can easily be expressed in terms of the function $S(z, a)$ (see [2]), and $S(z, a)$ is the solution to a simple Kerzman-Stein Fredholm integral equation of the second kind with $C^{\infty}$ kernel and inhomogeneous term (see $[2,3,9,12,13,16]$ ). The kernel $S(z, w)$ may be expressed as

$$
S(z, w)=\frac{c S(z, a) \overline{S(w, a)}}{1-f_{a}(z) \overline{f_{a}(w)}}
$$


where $c=1 / S(a, a)$. This shows that, once the boundary values of the single function of one variable $S(z, a)$ are known, the Szegö kernel can be evaluated at an arbitrary pair of points. A similar identity holds for the Bergman kernel,

$$
K(z, w)=\frac{4 \pi c^{2} S(z, a)^{2} \overline{S(w, a)^{2}}}{\left(1-f_{a}(z) \overline{f_{a}(w)}\right)^{2}}
$$

where $c=1 / S(a, a)$. This shows that the Bergman kernel is composed of the same basic functions that make up the Szegö kernel. Finally, the Poisson kernel $p(z, w)$ is given by

$$
p(z, w)=\frac{S(z, w) S(w, a)}{S(z, a)}+\frac{\overline{S(z, w) S(w, a) f_{a}(z)}}{\overline{S(z, a) f_{a}(w)}},
$$

where $z$ is a point in $\Omega$ and $w$ is a point in the boundary (see [2, page 37]). Thus, the Poisson kernel is also composed of the same basic functions. None of these formulas for the kernel functions in a simply connected domain could be considered very new. However, we shall prove analogous results for $n$-connected domains that are new. The new results show that there are $n+1$ basic functions that comprise all the kernels. An interesting feature of all the results in this paper is the central role played by the zeroes of the Szegö kernel.

The results of this paper were announced in [5]. A practical method for using the results of this paper to do numerical computations is given in [6].

2. The Ahlfors map and zeroes of the Szegö kernel. Before we start stating and proving our main theorems, we must review some basic facts about the kernel functions. Some of these facts are proved in Bergman's book [7]; all of them are proved in [2].

Suppose that $\Omega$ is a bounded $n$-connected domain in the plane with $C^{\infty}$ smooth boundary. Let $\gamma_{j}, j=1, \ldots, n$, denote the $n$ non-intersecting $C^{\infty}$ simple closed curves which define the boundary of $\Omega$, and suppose that $\gamma_{j}$ is parameterized in the standard sense by $z_{j}(t), 0 \leq t \leq 1$. We shall use the convention that $\gamma_{n}$ denotes the outer boundary curve of $\Omega$. Let $T(z)$ be the $C^{\infty}$ function defined on $b \Omega$ such that $T(z)$ is the complex number representing the unit tangent vector at $z \in b \Omega$ pointing in the direction of the standard orientation. This complex unit tangent vector function is characterized by the equation $T\left(z_{j}(t)\right)=z_{j}^{\prime}(t) /\left|z_{j}^{\prime}(t)\right|$.

We shall let $A^{\infty}(\Omega)$ denote the space of holomorphic functions on $\Omega$ that are in $C^{\infty}(\bar{\Omega})$. The space of complex valued functions on $\Omega$ that are square integrable with respect to Lebesgue area measure $d A$ will be written $L^{2}(\Omega)$, and the space of complex valued functions on $b \Omega$ that are square integrable with respect to arc length measure $d s$ will be denoted by $L^{2}(b \Omega)$. The Bergman 
space of holomorphic functions on $\Omega$ that are in $L^{2}(\Omega)$ shall be written $H^{2}(\Omega)$ and the Hardy space of functions in $L^{2}(b \Omega)$ that are the $L^{2}$ boundary values of holomorphic functions on $\Omega$ shall be written $H^{2}(b \Omega)$. The inner products associated to $L^{2}(\Omega)$ and $L^{2}(b \Omega)$ shall be written

$$
\langle u, v\rangle_{\Omega}=\iint_{\Omega} u \bar{v} d A \text { and }\langle u, v\rangle_{b \Omega}=\int_{b \Omega} u \bar{v} d s,
$$

respectively.

For each fixed point $a \in \Omega$, the Szegö kernel $S(z, a)$, as a function of $z$, extends to the boundary to be a function in $A^{\infty}(\Omega)$. Furthermore, $S(z, a)$ has exactly $(n-1)$ zeroes in $\Omega$ (counting multiplicities) and does not vanish at any points $z$ in the boundary of $\Omega$. We mention here that $S(z, w)$ is in $C^{\infty}((\bar{\Omega} \times \bar{\Omega})-\{(z, z): z \in b \Omega\})$ as a function of $(z, w)$, and that this well known fact will become evident from the formula for the kernel that we shall soon derive.

The Garabedian kernel $L(z, a)$ is a kernel related to the Szegö kernel via the identity

$$
\frac{1}{i} L(z, a) T(z)=S(a, z) \quad \text { for } z \in b \Omega \text { and } a \in \Omega .
$$

For fixed $a \in \Omega$, the kernel $L(z, a)$ is a holomorphic function of $z$ on $\Omega-\{a\}$ with a simple pole at $a$ with residue $1 /(2 \pi)$. Furthermore, as a function of $z$, $L(z, a)$ extends to the boundary and is in the space $C^{\infty}(\bar{\Omega}-\{a\})$. In fact, $L(z, a)$ extends to be in $C^{\infty}((\bar{\Omega} \times \bar{\Omega})-\{(z, z): z \in \bar{\Omega}\})$. Also, $L(z, a)$ is non-zero for all $(z, a)$ in $\bar{\Omega} \times \Omega$ with $z \neq a$.

The kernel $S(z, w)$ is holomorphic in $z$ and antiholomorphic in $w$ on $\Omega \times \Omega$, and $L(z, w)$ is holomorphic in both variables for $z, w \in \Omega, z \neq w$. We note here that $S(z, z)$ is real and positive for each $z \in \Omega$, and that $S(z, w)=\overline{S(w, z)}$ and $L(z, w)=-L(w, z)$. Also, the Szegő kernel reproduces holomorphic functions in the sense that

$$
h(a)=\langle h, S(\cdot, a)\rangle_{b \Omega}
$$

for all $h \in H^{2}(b \Omega)$ and $a \in \Omega$.

Given a point $a \in \Omega$, the Ahlfors map $f_{a}$ associated to the pair $(\Omega, a)$ is a proper holomorphic mapping of $\Omega$ onto the unit disc. It is an $n$-to-one mapping (counting multiplicities), it extends to be in $A^{\infty}(\Omega)$, and it maps each boundary curve $\gamma_{j}$ one-to-one onto the unit circle. Furthermore, $f_{a}(a)=0$, and $f_{a}$ is the unique function mapping $\Omega$ into the unit disc maximizing the quantity $\left|f_{a}^{\prime}(a)\right|$ with $f_{a}^{\prime}(a)>0$. The Ahlfors map is related to the Szegö kernel and Garabedian kernel via

$$
f_{a}(z)=\frac{S(z, a)}{L(z, a)}
$$


Note that $f_{a}^{\prime}(a)=2 \pi S(a, a) \neq 0$. Because $f_{a}$ is $n$-to-one, $f_{a}$ has $n$ zeroes. The simple pole of $L(z, a)$ at $a$ accounts for the simple zero of $f_{a}$ at $a$. The other $n-1$ zeroes of $f_{a}$ are given by $(n-1)$ zeroes of $S(z, a)$ in $\Omega-\{a\}$. Let $a_{1}, a_{2}, \ldots, a_{n-1}$ denote these $n-1$ zeroes (counted with multiplicity). I proved in [4] (see also [2, page 105]) that, if $a$ is close to one of the boundary curves, the zeroes $a_{1}, \ldots, a_{n-1}$ become distinct simple zeroes. It follows from this result that, for all but at most finitely many points $a \in \Omega, S(z, a)$ has $n-1$ distinct simple zeroes in $\Omega$ as a function of $z$.

3. A special orthonormal basis for the Hardy space. The zeroes of the Szegö kernel give rise to a particularly nice basis for the Hardy space of an $n$-connected domain with $C^{\infty}$ smooth boundary. We shall use the notation that we set up in the preceding section. We assume that $a \in \Omega$ is a fixed point in $\Omega$ that has been chosen so that the $n-1$ zeroes, $a_{1}, \ldots, a_{n-1}$, of $S(z, a)$ are distinct and simple. We shall let $a_{0}$ denote $a$ and we shall use the shorthand notation $f(z)$ for the Ahlfors map $f_{a}(z)$.

We shall now prove that the set of functions $\left\{h_{i k}(z): 0 \leq i \leq n-1\right.$ and $k \geq 0\}$, where $h_{i k}$ is defined via

$$
h_{i k}(z)=S\left(z, a_{i}\right) f(z)^{k},
$$

forms a basis for the Hardy space $H^{2}(b \Omega)$. Furthermore,

$$
\left\langle h_{i k}, h_{j m}\right\rangle_{b \Omega}= \begin{cases}0, & \text { if } k \neq m, \\ S\left(a_{j}, a_{i}\right), & \text { if } k=m .\end{cases}
$$

The proof of these assertions consists of three parts. First, we will prove that these functions span a dense subset of $H^{2}(b \Omega)$. Second, we will prove identity (3.1). Finally, we will show that identity (3.1) implies that the set is linearly independent. To prove the density of the span, suppose that $g \in H^{2}(b \Omega)$ is orthogonal to the span. Notice that the reproducing property of the Szegö kernel yields that

$$
\left\langle g, S\left(\cdot, a_{j}\right)\right\rangle_{b \Omega}=g\left(a_{j}\right),
$$

and, therefore, $g$ vanishes at $a_{0}, a_{1}, \ldots, a_{n-1}$. Suppose we have shown that $g$ vanishes to order $m$ at each $a_{j}, j=0,1, \ldots, n-1$. It follows that $g / f^{m}$ has removable singularities at each $a_{j}$ and so it can be viewed as an element of $H^{2}(b \Omega)$. The value of $g / f^{m}$ at $a_{j}$ is $(1 / m !) g^{(m)}\left(a_{j}\right) / f^{\prime}\left(a_{j}\right)^{m}$. Since $|f(z)|=1$ when $z \in b \Omega$, it follows that $1 / f(z)=\overline{f(z)}$ when $z \in b \Omega$, and we may write

$$
\left\langle g, S\left(\cdot, a_{j}\right) f^{m}\right\rangle_{b \Omega}=\left\langle\frac{g}{f^{m}}, S\left(\cdot, a_{j}\right)\right\rangle_{b \Omega}=\frac{1}{m !} \frac{g^{(m)}\left(a_{j}\right)}{f^{\prime}\left(a_{j}\right)^{m}} .
$$


(The last equality follows from the reproducing property of the Szegö kernel.) We conclude that $g$ vanishes to order $m+1$ at each $a_{j}$. By induction, $g$ vanishes to infinite order at each $a_{j}$ and hence, $g \equiv 0$. This proves the density. To prove (3.1), let us suppose first that $k>m$. The fact that $\bar{f}=1 / f$ on $b \Omega$ and the reproducing property of the Szegö kernel now yield that

$$
\begin{aligned}
\left\langle h_{i k}, h_{j m}\right\rangle_{b \Omega} & =\int_{z \in b \Omega} S\left(z, a_{i}\right) f(z)^{k-m} \overline{S\left(z, a_{j}\right)} d s \\
& =\int_{z \in b \Omega} S\left(a_{j}, z\right)\left[S\left(z, a_{i}\right) f(z)^{k-m}\right] d s \\
& =S\left(a_{j}, a_{i}\right) f\left(a_{j}\right)^{k-m} .
\end{aligned}
$$

The identity now follows because $f\left(a_{j}\right)=0$ for all $j$. If $k=m$, then

$$
\left\langle h_{i k}, h_{j m}\right\rangle_{b \Omega}=\int_{z \in b \Omega} S\left(a_{j}, z\right) S\left(z, a_{i}\right) d s=S\left(a_{j}, a_{i}\right),
$$

and identity (3.1) is proved. It is now easy to see that the functions $h_{i k}$ are linearly independent. Indeed, identity (3.1) reveals that we need only check that, for fixed $k$, the $n$ functions $h_{i k}, i=0,1, \ldots, n-1$, are independent, and this is true because a relation of the form

$$
\sum_{i=0}^{n-1} C_{i} S\left(z, a_{i}\right) \equiv 0
$$

implies, via the reproducing property of the Szegö kernel, that every function $g$ in the Hardy space satisfies

$$
\sum_{i=0}^{n-1} \overline{C_{i}} g\left(a_{i}\right)=0
$$

and it is easy to construct polynomials $g$ that violate such a condition.

We next orthonormalize the sequence $\left\{h_{i k}\right\}$ via the Gram-Schmidt procedure. Identity (3.1) shows that most of the functions in the sequence are already orthogonal, and so our task is quite easy. We need only fix $k$ and orthonormalize the $n$ functions $h_{i k}, i=0,1, \ldots, n-1$. We obtain an orthonormal set $\left\{H_{i k}\right\}$ given by

$$
H_{0 k}(z)=b_{00} S(z, a) f(z)^{k}
$$

and 


$$
H_{i k}(z)=\sum_{j=1}^{i} b_{i j} S\left(z, a_{j}\right) f(z)^{k}, \quad i=1, \ldots, n-1,
$$

where $b_{i i} \neq 0$ for each $i=0,1, \ldots, n-1$. Because $|f|=1$ on $b \Omega$, it follows that the coefficients $b_{i j}$ do not depend on $k$. Notice that $H_{i k}$ does not contain a term involving $S(z, a)$ if $i>0$, because of (3.1) and the fact that $S\left(a_{i}, a\right)=0$.

The Szegö kernel can be written in terms of our orthonormal basis via

$$
S(z, w)=\sum_{i=0}^{n-1} \sum_{k=0}^{\infty} H_{i k}(z) \overline{H_{i k}(w)} .
$$

The geometric sum

$$
\sum_{k=0}^{\infty} f(z)^{k} \overline{f(w)^{k}}=\frac{1}{1-f(z) \overline{f(w)}}
$$

can be factored from the expression for $S(z, w)$ to yield a formula like the one in the following theorem.

Theorem 3.1. The Szegö kernel can be evaluated at an arbitrary pair of points $(z, w)$ in $\Omega$ via the formula

$$
S(z, w)=\frac{1}{1-f(z) \overline{f(w)}}\left(c_{0} S(z, a) \overline{S(w, a)}+\sum_{i, j=1}^{n-1} c_{i j} S\left(z, a_{i}\right) \overline{S\left(w, a_{j}\right)}\right)
$$

where $f(z)$ denotes the Ahlfors map $f_{a}(z), c_{0}=1 / S(a, a)$, and the coefficients $c_{i j}$ are given as the coefficients of the inverse matrix to the matrix $\left[S\left(a_{j}, a_{k}\right)\right]$.

The only part of Theorem 3.1 that is unproved at the moment is the statement that identifies the coefficients in the formula. We have shown that these coefficients exist and that they are given as certain combinations of the GramSchmidt coefficients used above. That $c_{0}=1 / S(a, a)$ can be seen by setting $z=a$ and $w=a$ in (3.2). To complete the proof of Theorem 3.1, we shall now describe how to determine the coefficients $c_{i j}$. Suppose $1 \leq k \leq n-1$. Set $w=a_{k}$ in (3.2) and note that $f\left(a_{k}\right)=0$ and $S\left(a, a_{k}\right)=0$ to obtain

$$
S\left(z, a_{k}\right)=\sum_{i=1}^{n-1}\left(\sum_{j=1}^{n-1} c_{i j} S\left(a_{j}, a_{k}\right)\right) S\left(z, a_{i}\right) .
$$

We saw an identity like this when we showed above that the functions $h_{j k}$ are linearly independent for each fixed $k$. The same reasoning we used there yields that such a relation can only be true if

$$
\sum_{j=1}^{n-1} c_{i j} S\left(a_{j}, a_{k}\right)= \begin{cases}1, & \text { if } i=k \\ 0, & \text { if } i \neq k\end{cases}
$$


This shows that the $(n-1) \times(n-1)$ matrix $\left[S\left(a_{j}, a_{k}\right)\right]$ is invertible and that $\left[c_{i j}\right]$ is its inverse.

Theorem 3.1 generalizes in a routine manner to any domain $\Omega_{1}$ with nonsmooth boundary that can be mapped to a finitely connected domain with smooth boundary $\Omega_{2}$ via a biholomorphic mapping $\Phi$. The function $\Phi^{\prime}$ has a single valued holomorphic square root on $\Omega_{1}$ (see [2, page 43]) and the Szegö kernels transform under $\Phi$ via

$$
S_{1}(z, w)=\sqrt{\Phi^{\prime}(z)} S_{2}(\Phi(z), \Phi(w)) \overline{\sqrt{\Phi^{\prime}(w)}}
$$

and it is easy to see that the terms in (3.2) transform in exactly the correct manner in which to make (3.2) valid on $\Omega_{1}$.

We remark here that formula (3.2) shows that

$$
S(z, z)=\frac{\sigma(z)}{1-|f(z)|^{2}}
$$

where

$$
\sigma(z)=c_{0}|S(z, a)|^{2}+\sum_{i, j=1}^{n-1} c_{i j} S\left(z, a_{i}\right) S\left(a_{j}, z\right)
$$

is a non-vanishing function of $z$ in $C^{\infty}(\bar{\Omega})$. This shows that $1 / S(z, z)$ is a function in $C^{\infty}(\bar{\Omega})$ that vanishes on $b \Omega$ and has non-vanishing normal derivative on $b \Omega$. We shall need this fact later when we study the Poisson and Poisson-Szegö kernels.

4. Complexity of the Szegö kernel. Formula (3.2) reveals that the Szegö kernel associated to an $n$-connected domain is composed of the $n+1$ functions, $S(z, a), S\left(z, a_{1}\right), S\left(z, a_{2}\right), \ldots, S\left(z, a_{n-1}\right)$, and $L(z, a)$ (because $f(z)=$ $S(z, a) / L(z, a))$. Hence, the Szegö kernel is determined by the boundary values of these $n+1$ functions in the sense that the Szegö kernel may be evaluated at any pair of points $(z, w)$ in $\Omega \times \Omega$ by applying the Cauchy integral formula $2 n+2$ times $(n+1$ to evaluate the functions on the right hand side of (3.2) at $z$, and $n+1$ more to evaluate the functions at $w)$.

Kerzman and Stein [12] (see also $[2,3,9,13,16]$ ) proved that, on a smooth domain $\Omega$, the function $S_{a}(z)=S(z, a)$ is the solution to an explicit Fredholm integral equation of the second kind given by

$$
S_{a}(z)-\int_{w \in b \Omega} A(z, w) S_{a}(w) d s=\mathcal{C}_{a}(z)
$$


where $A(z, w)$ is the Kerzman-Stein kernel and $\mathcal{C}_{a}(z)$ is the Cauchy kernel. To be precise,

$$
A(z, w)=\frac{1}{2 \pi i}\left(\frac{T(w)}{w-z}-\frac{\overline{T(z)}}{\bar{w}-\bar{z}}\right)
$$

if $z, w \in b \Omega, z \neq w$, and $A(z, w)=0$ if $z=w$, and

$$
\mathcal{C}_{a}(z)=\frac{1}{2 \pi i} \frac{\overline{T(z)}}{\bar{a}-\bar{z}}
$$

The Kerzman-Stein kernel is skew-hermitian and, in spite of the apparent singularity at $z=w$ in the formula above, it is in $C^{\infty}(b \Omega \times b \Omega)$. (Kerzman and Stein discovered that the apparent singularities in the formula for $A(z, w)$ exactly cancel.) The Cauchy kernel is in $C^{\infty}(b \Omega)$. It follows from standard theory that this integral equation has a unique $C^{\infty}$ smooth solution. (See Kerzman and Trummer [16] and [3, 9] for descriptions of convenient ways to write and to solve this integral equation.)

The Kerzman-Stein equation produces the boundary values of $S(z, a)$. The boundary values of the Garabedian kernel $L(z, a)$ are then determined via identity (2.1), and the boundary values of the Ahlfors map $f_{a}(z)$ can now be gotten from (2.2). The remaining functions in (3.2) can be computed via the KerzmanStein integral equation once the zeroes $a_{1}, \ldots, a_{n-1}$ of $S_{a}$ have been located.

5. The Bergman kernel. In this section, we shall prove that the Bergman kernel of an $n$-connected domain in the plane with $C^{\infty}$ smooth boundary is composed of the same basic functions that comprise the Szegö kernel. It will follow that the Bergman kernel can be computed at every pair of points by solving $n$ one dimensional Fredholm integral equations of the second kind, and that at no point is it necessary to evaluate a double integral with respect to area measure.

The Bergman kernel $K(z, w)$ is related to the Szegő kernel via the identity

$$
K(z, w)=4 \pi S(z, w)^{2}+\sum_{i, j=1}^{n-1} A_{i j} F_{i}^{\prime}(z) \overline{F_{j}^{\prime}(w)}
$$

where the functions $F_{i}^{\prime}(z)$ are classical functions of potential theory described as follows. The harmonic function $\omega_{j}$ which solves the Dirichlet problem on $\Omega$ with boundary data equal to one on the boundary curve $\gamma_{j}$ and zero on $\gamma_{k}$ if $k \neq j$ has a multivalued harmonic conjugate. The function $F_{j}^{\prime}(z)$ is a globally defined single valued holomorphic function on $\Omega$ which is locally defined as the derivative of $\omega_{j}+i v$ where $v$ is a local harmonic conjugate for $\omega_{j}$. The Cauchy-Riemann equations reveal that $F_{j}^{\prime}(z)=2\left(\partial \omega_{j} / \partial z\right)$. 
Let $\mathcal{F}^{\prime}$ denote the vector space of functions given by the complex linear span of the set of functions $\left\{F_{j}^{\prime}(z): j=1, \ldots, n-1\right\}$. It is a classical fact that $\mathcal{F}^{\prime}$ is $n-1$ dimensional. Notice that $S\left(z, a_{i}\right) L(z, a)$ is in $A^{\infty}(\Omega)$ because the pole of $L(z, a)$ at $z=a$ is cancelled by the zero of $S\left(z, a_{i}\right)$ at $z=a$. A theorem due to Schiffer (see $[14,2,4])$ states that the $n-1$ functions $S\left(z, a_{i}\right) L(z, a)$, $i=1, \ldots, n-1$, form a basis for $\mathcal{F}^{\prime}$. We may now write

$$
K(z, w)=4 \pi S(z, w)^{2}+\sum_{i, j=1}^{n-1} \lambda_{i j} S\left(z, a_{i}\right) L(z, a) \overline{S\left(w, a_{j}\right) L(w, a)}
$$

which, together with (3.2) allows us to write down a formula which sheds light on the degree of complexity of the Bergman kernel.

Theorem 5.1. The Bergman kernel is composed of the same basic functions that make up the Szegö kernel, as evidenced by the following formula.

$$
\begin{gathered}
K(z, w)=\frac{1}{(1-f(z) \overline{f(w)})^{2}}\left(\sum_{\substack{0 \leq i \leq j \leq n-1 \\
0 \leq k \leq m \leq n-1}} C_{i j k m} S\left(z, a_{i}\right) S\left(z, a_{j}\right) \overline{S\left(w, a_{k}\right) S\left(w, a_{m}\right)}\right) \\
+\sum_{i, j=1}^{n-1} \lambda_{i j} S\left(z, a_{i}\right) L(z, a) \overline{S\left(w, a_{j}\right) L(w, a)} .
\end{gathered}
$$

A recipe is given for explicitly computing all the coefficients appearing in the formula for the Bergman kernel in Theorem 5.1 in my paper [6]. It is interesting that all the elements of the kernel function can be computed by means of one dimensional line integrals and simple linear algebra.

We have shown that the formula in Theorem 5.1 is valid on a domain with smooth boundary. If a finitely connected domain $\Omega_{1}$ does not have smooth boundary, and if none of its boundary components are points, there is a conformal mapping $f$ of $\Omega_{1}$ onto a domain $\Omega_{2}$ whose boundary is smooth. The transformation formula for the Bergman kernels under biholomorphic mappings,

$$
K_{1}(z, w)=f^{\prime}(z) K_{2}(f(z), f(w)) \overline{f^{\prime}(w)}
$$

together with the transformation formula for the Szegö kernels (3.3), can then be used to show that the formula in Theorem 5.1 is valid on $\Omega_{1}$. We thereby obtain the following theorem, which shows that the Bergman kernel of a finitely connected domain can never be a genuine function of two complex variables. 
Theorem 5.2. Suppose $\Omega$ is a finitely connected domain such that no boundary component of $\Omega$ is a point. Let $f(z)$ denote an Ahlfors map of $\Omega$ onto the unit disc. The Bergman kernel $K(z, w)$ associated to $\Omega$ is a function of the form

$$
K(z, w)=\frac{1}{(1-f(z) \overline{f(w)})^{2}}\left(\sum_{j, k=1}^{n(n+1) / 2} C_{j k} H_{j}(z) \overline{H_{k}(w)}\right)+\sum_{i, j=1}^{n-1} \lambda_{i j} G_{i}(z) \overline{G_{j}(w)}
$$

where the functions $H_{j}$ and $G_{j}$ are functions of one variable in the Bergman space.

6. Characterization of domains with rational kernel functions. In the previous sections, we have shown that the kernel functions are not as complex as one might expect them to be. In this section, we shall prove theorems that say, roughly, that the only domain whose Bergman or Szegó kernel is so simple as to be rational is the disc.

A function $R(z, w)$ of two complex variables is called rational if there are relatively prime polynomials $P(z, w)$ and $Q(z, w)$ such that

$$
R(z, w)=\frac{P(z, w)}{Q(z, w)} .
$$

It is not hard to prove that a function $H(z, w)$ which is holomorphic in $z$ and $w$ on a product domain $\Omega_{1} \times \Omega_{2}$ is rational if and only if, for each fixed $b \in \Omega_{2}$, the function $H(z, b)$ is rational in $z$, and for each fixed $a \in \Omega_{1}$, the function $H(a, w)$ is rational in $w$ (see Bochner and Martin [8, page 201]). We shall say that the Bergman kernel function $K(z, w)$ associated to a domain $\Omega$ is rational if it can be written as $R(z, \bar{w})$ where $R$ is a holomorphic rational function of two variables. Because the Bergman kernel is hermitian, the facts above imply that $K(z, w)$ is rational if and only if, for each point $a \in \Omega$, the function $K(z, a)$ is a rational function of $z$. In fact, $K(z, w)$ is rational if and only if there exists a small disc $D_{\varepsilon}\left(w_{0}\right) \subset \Omega$ such that $K(z, a)$ is a rational function of $z$ for each $a \in D_{\varepsilon}\left(w_{0}\right)$. Similar statements hold for the other kernel functions.

Theorem 6.1. Suppose $\Omega$ is a bounded $n$-connected domain, $n>1$, with $C^{\infty}$ smooth boundary. Neither the Bergman kernel nor the Szegö kernel associated to $\Omega$ can be rational functions.

The assumption in Theorem 6.1 that the boundary of $\Omega$ is $C^{\infty}$ smooth can be relaxed. For example, the conclusion about the Szego" kernel holds if the boundary is only assumed to be $C^{2}$ smooth. The conclusion about the Bergman kernel holds if the domain is only assumed to be finitely connected and such that 
no boundary component is a point. We shall explain how to relax the smoothness assumptions later in this section.

Before we proceed to prove Theorem 6.1, let us consider the case of a oneconnected domain $\Omega \neq \mathbb{C}$. If $f_{a}$ is a Riemann mapping $f_{a}: \Omega \rightarrow D_{1}(0)$ such that $f_{a}(a)=0$ and $f_{a}^{\prime}(a)>0$, the Bergman kernel for $\Omega$ can be expressed via

$$
K(z, w)=\frac{f_{a}^{\prime}(z) \overline{f_{a}^{\prime}(w)}}{\pi\left(1-f_{a}(z) \overline{f_{a}(w)}\right)^{2}} .
$$

If we set $w=a$ in this formula, we obtain the identity

$$
K(z, a)=C f_{a}^{\prime}(z)
$$

where $C=f_{a}^{\prime}(a) / \pi$ is a positive constant. If we differentiate the formula with respect to $\bar{w}$ and then set $w=a$, we obtain

$$
\frac{\partial}{\partial \bar{w}} K(z, a)=f_{a}^{\prime}(z)\left(C_{1}+C_{2} f_{a}(z)\right)
$$

where $C_{1}$ and $C_{2}$ are constants, and $C_{2} \neq 0$. (In fact, $C_{2}=2 f_{a}^{\prime}(a)^{2} / \pi$.) It can easily be deduced from these formulas that the Bergman kernel is rational if and only if the Riemann map is rational.

To study the Szegö kernel, assume that $\Omega$ is a bounded simply connected domain with $C^{2}$ smooth boundary and let $f_{a}$ denote a Riemann map as above. The Szegö kernel is given by

$$
S(z, w)=\frac{\sqrt{f_{a}^{\prime}(z)} \overline{\sqrt{f_{a}^{\prime}(w)}}}{2 \pi\left(1-f_{a}(z) \overline{f_{a}(w)}\right)} .
$$

Set $w=a$ in this formula to obtain

$$
S(z, a)=c \sqrt{f_{a}^{\prime}(z)}
$$

where $c=\sqrt{f_{a}^{\prime}(a)} /(2 \pi)$ is a positive constant. Now differentiate the formula with respect to $\bar{w}$ and then set $w=a$ to obtain

$$
\frac{\partial}{\partial \bar{w}} S(z, a)=\sqrt{f_{a}^{\prime}(z)}\left(c_{1}+c_{2} f_{a}(z)\right)
$$

where $c_{1}$ and $c_{2}$ are constants, and $c_{2} \neq 0$. (In fact, $c_{2}=f_{a}^{\prime}(a)^{3 / 2} /(2 \pi)$.) These formulas reveal that the Szegó kernel is rational if and only if the Riemann map and the square root of its derivative are rational. Let us summarize these results in the following theorem. 
Theorem 6.2. Suppose $\Omega \neq \mathbb{C}$ is a simply connected domain. The Bergman kernel associated to $\Omega$ is rational if and only if there is a rational biholomorphic mapping $f(z)$ mapping $\Omega$ one-to-one onto the unit disc. If $\Omega$ is further assumed to be bounded and have $C^{2}$ smooth boundary, then the Szego" kernel associated to $\Omega$ is rational if and only if there is a rational biholomorphic mapping $f(z)$ mapping $\Omega$ one-to-one onto the unit disc such that $f^{\prime}(z)$ is the square of a rational function.

Proof of Theorem 6.1. We shall use the notation that we set up previously to describe our $n$-connected domain $\Omega$. Hence, $\gamma_{n}$ denotes the outer boundary of $\Omega$. Since we are assuming that $n>1$, we may let $\gamma_{1}$ denote one of the inner boundary curves of $\Omega$, and we let $D_{1}$ denote the bounded region enclosed by $\gamma_{1}$.

We first assume that the Szegö kernel associated to $\Omega$ is rational. Formula (3.2) shows that it then follows that the Ahlfors mapping $f_{a}(z)$ is a rational function of $z$ for each point $a$ in $\Omega$, minus the finite set where the zeroes of $S(z, a)$ might not all be simple zeroes. (It was proved earlier by M. Jeong [10, 11], using other techniques, that the Ahlfors maps are all rational.) We may now use formula (2.2) to deduce that the Garabedian kernel $L(z, a)$ is a rational function of $z$ for each $a$ in an open subset of $\Omega$, and hence that $L(z, a)$ is a rational function of $(z, a)$. It is clear that the Ahlfors maps $f_{a}$ can have no poles on $b \Omega$. Since the boundary of $\Omega$ is assumed to be smooth, the Hopf lemma implies that $f_{a}^{\prime}(z) \neq 0$ for $z \in b \Omega$. Since the boundary curves of $\Omega$ are described by the equation $\left|f_{a}(z)\right|=1$, it follows that the boundary curves of $\Omega$ are all real analytic curves. From this it follows that $S(z, w)$ extends holomorphically in $z$ and antiholomorphically in $w$ to an open set in $\mathbb{C} \times \mathbb{C}$ containing $\bar{\Omega} \times \bar{\Omega}-\{(z, z)$ : $z \in b \Omega\}$, and that $L(z, w)$ extends holomorphically in $z$ and $w$ to an open set in $\mathbb{C} \times \mathbb{C}$ containing $\bar{\Omega} \times \bar{\Omega}-\{(z, z): z \in \bar{\Omega}\}$. This may seem like a silly thing to say in light of the fact that $S(z, w)$ and $L(z, w)$ are rational, however it implies that singularities must stay away from $b \Omega \times b \Omega-\{(z, z): z \in b \Omega\}$.

We shall be concerned with the number of zeroes and poles of $S(z, a)$ and $L(z, a)$ as functions of $z$ which lie in $D_{1}$, and we shall consider how these numbers vary as $a$ moves from a point on the outer boundary of $\Omega$ to a point on $\gamma_{1}$. First, however, we shall need to review some properties of the zeroes of the Szegö kernel proved in [4] (see also [2]). We mentioned earlier that if $a \in \Omega$, then $S(z, a) \neq 0$ and $L(z, a) \neq 0$ for all $z \in b \Omega$. We also mentioned that neither $S(z, a)$ nor $L(z, a)$ can have poles on $b \Omega$. We shall use these facts to see that zeroes and poles of $S(z, a)$ and $L(z, a)$ which lie in $D_{1}$ cannot exit $D_{1}$ through $\gamma_{1}$ as $a$ varies in $\Omega$. We also mentioned earlier that $S(z, a)$ has $n-1$ zeroes in $\Omega$ as a function of $z$, and $L(z, a) \neq 0$ for all $z \in \bar{\Omega}-\{a\}$. It is proved in [4] that, if $a \in \Omega$ is allowed to tend to a point $A_{k}$ in a boundary curve $\gamma_{k}$, then the $n-1$ zeroes of $S(z, a)$ separate into simple zeroes which migrate to distinct points on the boundary in such a 
way that there is a point on each boundary curve $\gamma_{j}, j \neq k$, to which exactly one of the zeroes tends. To be precise, there exist points $\left\{A_{j}: 1 \leq j \leq n, j \neq k\right\}$ with $A_{j} \in \gamma_{j}$ such that the $n-1$ zeroes of $S(z, a)$ can be listed as $a_{j}, 1 \leq j \leq n$, $j \neq k$, where $a_{j}$ tends to $A_{j}$ for each $j \neq k$ as $a$ tends to $A_{k}$.

Since $S(z, w)$ is rational, there exist relatively prime polynomials $P(z, w)$ and $Q(z, w)$ such that $S(z, w)=P(z, \bar{w}) / Q(z, \bar{w})$. There are at most finitely many points $w_{0} \in \mathbb{C}$ for which the equations $P\left(z, \bar{w}_{0}\right)=0$ and $Q\left(z, \bar{w}_{0}\right)=0$ have a common root (see Ahlfors [1, page 300]). Let $B_{S}$ denote the (possibly empty) set of such points $w_{0}$. Similarly, there are relatively prime polynomials $p(z, w)$ and $q(z, w)$ such that $L(z, w)=p(z, w) / q(z, w)$, and there is a finite set $B_{L}$ of points $w_{0}$ where the equations $p\left(z, w_{0}\right)=0$ and $q\left(z, w_{0}\right)=0$ have a common root. Let $B=B_{S} \cup B_{L}$.

Let $S_{a}(z)=S(z, a)$. It is a simple exercise using the argument principle that the zeroes and poles of $S_{a}$ are continuous functions of $a$ when $a \notin B$ in the following sense. Suppose $z_{0}$ is a zero of multiplicity $m$ of $S\left(z, a_{0}\right)$ where $a_{0} \notin B$. Given $\varepsilon>0$ such that $z_{0}$ is the only zero of $S\left(z, a_{0}\right)$ in $\overline{D_{\varepsilon}\left(z_{0}\right)}$, there is a $\delta>0$ such that $S(z, a)$ has precisely $m$ zeroes in $\overline{D_{\varepsilon}\left(z_{0}\right)}$ as a function of $z$ (counting multiplicities) when $a \in D_{\delta}\left(a_{0}\right)$. A similar statement holds for poles of $S(z, a)$, and for zeroes and poles of $L(z, a)$.

We have stated all the necessary facts to be able to assert that there exist non-negative integers $Z_{S}, Z_{L}, P_{S}$, and $P_{L}$ such that, for any point $a \in \Omega-B$, $Z_{S}$ is equal to the number of zeroes of $S(z, a)$ in $\overline{D_{1}}, Z_{L}$ is equal to the number of zeroes of $L(z, a)$ in $\overline{D_{1}}, P_{S}$ is equal to the number of poles of $S(z, a)$ in $\overline{D_{1}}$, and $P_{L}$ is equal to the number of poles of $L(z, a)$ in $\overline{D_{1}}$.

Let $\sigma$ denote a curve in $\bar{\Omega}-B$ which starts at a point $A_{n}$ on the outer boundary $\gamma_{n}$ of $\Omega$, travels through $\Omega$, and terminates at a point $A_{1}$ in $\gamma_{1}$. We shall be able to deduce relationships between the four integers, $Z_{S}, Z_{L}, P_{S}$, and $P_{L}$, by letting $a$ tend to the two endpoints of $\sigma$. The relationships shall turn out to be contradictory. To find relationships between these numbers, we shall need to use an argument from [4]. Since the boundary curves of $\Omega$ are real analytic curves, there exists an antiholomorphic reflection function $R(z)$ with the properties that $R(z)$ is defined and is antiholomorphic on a neighborhood $\mathcal{O}$ of $b \Omega, R\left(z_{0}\right)=z_{0}$ when $z_{0} \in b \Omega, R^{\prime}(z)$ is non-vanishing on $\mathcal{O}$, and $R(z)$ maps $\mathcal{O} \cap \Omega$ one-to-one onto $\mathcal{O}-\bar{\Omega}$.

Let $w_{k}$ be a sequence of points in $\Omega$ that tend to $A_{n}$ along $\sigma$, and let $a$ be a fixed point in $\Omega-B$. By (2.1), we have $-i L(z, a) T(z)=S(a, z)$ and $-i L\left(z, w_{k}\right) T(z)=S\left(w_{k}, z\right)$ for $z \in b \Omega$. Divide the second of these identities by the first and use the fact that $R(z)=z$ on $b \Omega$ to obtain

$$
\frac{S\left(w_{k}, z\right)}{S(a, z)}=\frac{L\left(R(z), w_{k}\right)}{L(R(z), a)} \quad \text { for } z \in b \Omega \text {. }
$$


The function on the left hand side of (6.1) is antiholomorphic in $z$ on a neighborhood of $b \Omega$; so is the function on the right hand side. Since these functions agree on $b \Omega$, they must be equal on a neighborhood of $b \Omega$. In fact, because $S$ and $L$ are rational, these two functions are equal as meromorphic functions on the neighborhood $\mathcal{O}$ of $b \Omega$ on which $R(z)$ is defined. We may assume that $\mathcal{O}$ is small enough that $S(z, a)$ and $L(z, a)$ have no poles or zeroes in $\mathcal{O}$. Formula (6.1) now allows us to read off the following facts (keep in mind that $w_{k}$ is close to $\left.A_{n} \in \gamma_{n}\right)$. If $S\left(z, w_{k}\right)$ has a zero $z_{0} \in \Omega$ near $\gamma_{1}$, then $L\left(R\left(z_{0}\right), w_{k}\right)=0$, i.e., $L\left(z, w_{k}\right)$ has a zero at the reflected point $R\left(z_{0}\right) \in D_{1}$ near $b \Omega$. Neither $S\left(z, w_{k}\right)$ nor $L\left(z, w_{k}\right)$ can have a pole $z_{0} \in D_{1}$ near $\gamma_{1}$ because neither $L\left(z, w_{k}\right)$ nor $S\left(z, w_{k}\right)$ has a pole at the reflected point $R\left(z_{0}\right) \in \Omega$.

Finally, notice that (2.1) yields that

$$
-i L\left(A_{n}, z\right) T\left(A_{n}\right)=S\left(z, A_{n}\right) \quad \text { for } z \in \Omega,
$$

and consequently $-i L\left(A_{n}, z\right) T\left(A_{n}\right)=S\left(z, A_{n}\right)$ for $z \in \overline{D_{j}}$. Hence, the functions $L\left(A_{n}, z\right)$ and $S\left(z, A_{n}\right)$ have the same number of zeroes and poles in $\overline{D_{j}}$. It is proved in [4] that $S\left(z, A_{n}\right)$ has a single simple zero on $\gamma_{1}$ and this zero is approached by single simple zeroes of $S\left(z, w_{k}\right)$ as $k \rightarrow \infty$. No other zeroes of $S\left(z, w_{k}\right)$ can migrate near $\gamma_{1}$. Our remarks above yield that $L\left(z, w_{k}\right)$ has a simple zero at the reflection of the zero of $S\left(z, w_{k}\right)$ near $\gamma_{1}$. By letting $k \rightarrow \infty$, we obtain the relations

$$
Z_{L}=Z_{S}+1, \quad P_{L}=P_{S}
$$

We now take a sequence of points $w_{k}$ in $\Omega$ that tend to $A_{1}$ along $\sigma$. Formula (6.1) remains valid and, if we reason as above, we deduce that, since $S\left(z, w_{k}\right)$ has no zeroes $z_{0}$ with $z_{0}$ near $\gamma_{1}, L\left(z, w_{k}\right)$ has no zeroes in $D_{j}$ near $\gamma_{1}$. However, since $L\left(z, w_{k}\right)$ has a simple pole at $z=w_{k}$, it follows that $S\left(z, w_{k}\right)$ has a simple pole at the reflected point $R\left(w_{k}\right)$ in $D_{1}$. We now let $k \rightarrow \infty$ and use the facts that $S\left(z, A_{1}\right)$ and $L\left(z, A_{1}\right)$ have the same zeroes and poles in $\overline{D_{1}}$ and that one of those poles is a simple pole at $A_{1}$ to obtain

$$
Z_{L}=Z_{S}, \quad P_{L}+1=P_{S} .
$$

These relationships contradict the ones we obtained by letting $w_{k}$ tend to $A_{n}$, and we conclude that $\Omega$ cannot be multiply connected.

We now turn to the study of the Bergman kernel. Assume that $K(z, w)$ is rational. We shall use an argument similar to the one above for the Szegö kernel, however, many of the underlying facts are different. Before we can begin, we must review some facts about the Bergman kernel (see [2] for proofs of these facts). 
We first must prove that if the Bergman kernel associated to a bounded domain is rational, then any proper holomorphic mapping of the domain onto the unit disc must be rational. Suppose $f: \Omega \rightarrow D_{1}(0)$ is a proper holomorphic map. Such a map must be in $A^{\infty}(\Omega)$ and there is a positive integer $m$ such that $f$ is an $m$-to-one mapping of $\Omega$ onto $D_{1}(0)$ (see [2, page $\left.62-70\right]$ ). The branch locus $\mathcal{B}=\left\{z \in \Omega: f^{\prime}(z)=0\right\}$ is a finite set, and for each point $w_{0}$ in $D_{1}(0)-f(\mathcal{B})$, there are exactly $m$ distinct points in $f^{-1}\left(w_{0}\right)$. Near such a point $w_{0}$, there is an $\varepsilon>0$ such that it is possible to define $m$ holomorphic maps $F_{1}(w), \ldots, F_{m}(w)$ on $D_{\varepsilon}\left(w_{0}\right)$, which map into $\Omega-\mathcal{B}$ such that $f\left(F_{k}(w)\right)=w$. These local inverses appear in the following transformation formula for the Bergman kernels under a proper holomorphic mapping. Let $K_{1}(z, w)=\pi^{-1}(1-z \bar{w})^{-2}$ denote the Bergman kernel of the unit disc (and recall that $K(z, w)$ denotes the Bergman kernel for $\Omega$ ). It is proved in [2, page 68] that the kernels transform via

$$
f^{\prime}(z) K_{1}(f(z), w)=\sum_{k=1}^{m} K\left(z, F_{k}(w)\right) \overline{F_{k}^{\prime}(w)}
$$

Although the functions $F_{k}$ are only locally defined on $D_{1}(0)-f(\mathcal{B})$, the function on the right hand side of the transformation formula, being symmetric in the $F_{k}$, is globally well defined. In fact, the function is holomorphic in $z$ and antiholomorphic in $w$ for $(z, w) \in \Omega \times\left(D_{1}(0)-f(\mathcal{B})\right)$. (The set $f(\mathcal{B})$ can be seen to be a removable singularity set, but we shall not need to know this.) If the origin is in $D_{1}(0)-f(\mathcal{B})$, we replace $f$ by its composition with a Möbius transformation so that $0 \notin D_{1}(0)-f(\mathcal{B})$. We now set $w=0$ in the transformation formula for the Bergman kernels to obtain

$$
f^{\prime}(z)=\pi \sum_{k=1}^{m} K\left(z, F_{k}(0)\right) \overline{F_{k}^{\prime}(0)}
$$

This shows that $f^{\prime}(z)$ is a rational function. Now differentiate the transformation formula with respect to $\bar{w}$ and then set $w=0$ to obtain

$$
2 f^{\prime}(z) f(z)=\pi \sum_{k=1}^{m} \frac{\partial}{\partial \bar{w}} K\left(z, F_{k}(0)\right) \overline{F_{k}^{\prime}(0)^{2}}+\pi \sum_{k=1}^{m} K\left(z, F_{k}(0)\right) \overline{F_{k}^{\prime \prime}(0)} .
$$

We may now deduce that $f^{\prime}(z) f(z)$ is rational, and so it follows that $f(z)$ is rational.

Since the Ahlfors mappings $f_{a}(z)$ are proper mappings of $\Omega$ onto the unit disc, they are rational functions of $z$. As above, this implies that the boundary curves of $\Omega$ are all real analytic curves, and from this it follows that $K(z, w)$ 
extends holomorphically in $z$ and antiholomorphically in $w$ to an open set in $\mathbb{C} \times \mathbb{C}$ containing $\bar{\Omega} \times \bar{\Omega}-\{(z, z): z \in b \Omega\}$.

The Bergman kernel is related to the classical Green's function via

$$
K(z, w)=-\frac{2}{\pi} \frac{\partial^{2} G(z, w)}{\partial z \partial \bar{w}} .
$$

Define another function $\Lambda(z, w)$ on $\Omega$ via

$$
\Lambda(z, w)=-\frac{2}{\pi} \frac{\partial^{2} G(z, w)}{\partial z \partial w}
$$

(This function is sometimes written $L(z, w)$ in the literature; we have chosen the symbol $\Lambda$ here to avoid confusion with our notation for the Garabedian kernel above.) It follows from known properties of the Green's function that $\Lambda(z, w)$ extends holomorphically in $z$ and $w$ to an open set in $\mathbb{C} \times \mathbb{C}$ containing $\bar{\Omega} \times \bar{\Omega}-\{(z, z): z \in \bar{\Omega}\}$, and that, if $a \in \bar{\Omega}$, then $\Lambda(z, a)$ has a double pole at $z=a$ as a function of $z$.

We shall need to use the following real variable theorem. Suppose that $R(x, y)$ is a real analytic function of $(x, y)$ on a product domain $U_{1} \times U_{2} \subset$ $\mathbb{R}^{n} \times \mathbb{R}^{m}$ such that $R\left(x, y_{0}\right)$ is a rational function of $x$ on $U_{1}$ for each $y_{0} \in U_{2}$, and $R\left(x_{0}, y\right)$ is a rational function of $y$ on $U_{2}$ for each $x_{0} \in U_{1}$. It then follows that $R(x, y)$ is a rational function of $(x, y)$ (the proof in Bochner and Martin [8, page 201] works in the real case, too).

We now consider the function $\left|f_{a}(z)\right|^{2}=|S(z, a)|^{2} /|L(z, a)|^{2}$. We know that for each fixed $a \in \Omega$, the function $f_{a}(z)$ is a rational function of $z$, and hence $\left|f_{a}(z)\right|^{2}$ is a rational function of $(x, y)$ where $z=x+i y$. Since $\left|f_{a}(z)\right|=\left|f_{z}(a)\right|$, the real variable theorem mentioned above implies that $\left|f_{a}(z)\right|^{2}$ is a rational function of the variables $\operatorname{Re} z, \operatorname{Im} z, \operatorname{Re} a$, and $\operatorname{Im} a$.

Assume that $a \in \Omega$ is such that the zeroes of $S(z, a)$ are all simple zeroes. Because Ahlfors maps are proper holomorphic maps, it is easy to verify that

$$
\frac{1}{2} \ln \left|f_{a}(z)\right|^{2}=G(z, a)+\sum_{i=1}^{n-1} G\left(z, a_{i}\right)
$$

where the points $a_{i}, i=1, \ldots, n-1$ are the zeroes of $S(z, a)$ (which, together with $a$, are the zeroes of $f_{a}$ ). We now consider the way in which the zeroes $a_{i}$ depend on $a$, and we write $a_{i}(a)$ in order to regard $a_{i}$ as a function of $a$. Let $A_{0}$ be a fixed point in $\Omega$ such that the zeroes of $S\left(z, A_{0}\right)$ are simple. Since the points $a_{i}\left(A_{0}\right)$ are distinct, we may choose an $\varepsilon>0$ such that $\overline{D_{\varepsilon}\left(a_{i}\left(A_{0}\right)\right)} \subset \Omega$ for each $i$ and $\overline{D_{\varepsilon}\left(a_{i}\left(A_{0}\right)\right)} \cap \overline{D_{\varepsilon}\left(a_{j}\left(A_{0}\right)\right)}=\varnothing$ if $i \neq j$. Thus, $a_{i}\left(A_{0}\right)$ is the only 
zero of $S\left(z, A_{0}\right)$ in $\overline{D_{\varepsilon}\left(a_{i}\left(A_{0}\right)\right)}$. The dependence of the zeroes of $S(z, a)$ on $a$ can be described by the formula,

$$
a_{i}(a)=\frac{1}{2 \pi i} \int_{\left|z-a_{i}\left(A_{0}\right)\right|=\varepsilon} z \frac{\frac{\partial}{\partial z} S(z, a)}{S(z, a)} d z,
$$

which is valid when $a$ is close to $A_{0}$. Because $S(z, a)$ is antiholomorphic in $a$, this formula shows that $a_{i}(a)$ is an antiholomorphic function of $a$ near $A_{0}$. We now differentiate (6.2) with respect to $z$ to obtain

$$
\frac{f_{a}^{\prime}(z)}{2 f_{a}(z)}=\frac{\partial}{\partial z} G(z, a)+\sum_{i=1}^{n-1} \frac{\partial}{\partial z} G\left(z, a_{i}\right) .
$$

Next, we differentiate with respect to $a$ and use the complex chain rule to obtain

$$
\frac{\partial}{\partial a}\left(\frac{f_{a}^{\prime}(z)}{2 f_{a}(z)}\right)=\frac{\partial^{2} G(z, a)}{\partial z \partial a}+\sum_{i=1}^{n-1} \frac{\partial^{2} G\left(z, a_{i}\right)}{\partial z \partial \bar{a}_{i}} \frac{\partial \bar{a}_{i}}{\partial a} .
$$

We now claim that the function on the left hand side of (6.3) is is a rational function $R(z, a)$ of $z$ and $a$. Indeed, because $\left|f_{a}(z)\right|^{2}$ is rational in the real and imaginary parts of $z$ and $a$, it follows that $R(z, a)$ is rational in the real and imaginary parts of $z$ and $a$. It is clear that $R(z, a)$ is holomorphic in $z$. Since $\left|f_{a}(z)\right|=\left|f_{z}(a)\right|$, it follows that $R(z, a)=R(a, z)$, and so $R(z, a)$ is holomorphic in $a$, too. Consequently, $R(z, a)$ is a rational function of $z$ and $a$. The function on the right hand side of (6.3) can be rewritten to yield

$$
R(z, a)=-\frac{\pi}{2} \Lambda(z, a)-\frac{\pi}{2} \sum_{i=1}^{n-1} K\left(z, a_{i}\right) \frac{\partial \bar{a}_{i}}{\partial a} .
$$

This last formula shows that, for each fixed $a$ in an open subset of $\Omega$, the function $\Lambda(z, a)$ is a rational function of $z$. Since $\Lambda(z, a)=\Lambda(a, z)$, we conclude that $\Lambda(z, a)$ is a rational function of $(z, a)$.

The Bergman kernel is related to $\Lambda$ via the identity

$$
\Lambda(w, z) T(z)=-K(w, z) \overline{T(z)} \quad \text { for } w \in \Omega \text { and } z \in b \Omega
$$

(see [2, page 135]). Define the set $B$ to be the finite set of points $a$ at which the numerators and denominators of $K(z, a)$ and $\Lambda(z, a)$ have common zeroes as functions of $z$. Let $\sigma$ denote a curve in $\bar{\Omega}-B$ which starts at a point $A_{n}$ on the outer boundary $\gamma_{n}$ of $\Omega$, travels through $\Omega$, and terminates at a point $A_{1}$ 
on an inner boundary curve $\gamma_{1}$. Since $K(z, a)$ and $\Lambda(z, a)$ cannot have poles on the boundary as functions of $z$ when $a \in \Omega$, the number $P_{K}$ of poles of $K(z, a)$ as a function of $z$ which lie in $D_{1}$ is constant as a moves along $\sigma$ away from the endpoints of the curve. Also, the number $P_{\Lambda}$ of poles of $\Lambda(z, a)$ in $D_{1}$ is constant as $a$ moves along the curve. We shall deduce relationships between $P_{K}$ and $P_{\Lambda}$ by letting $a$ tend to the two endpoints of $\sigma$. The relationships shall turn out to be contradictory.

Let $w_{k}$ be a sequence of points in $\Omega$ that tend to $A_{n}$ along $\sigma$. Since $K(z, a)$ and $\Lambda(z, a)$ cannot vanish for $z \in b \Omega$ when $a$ is close to the boundary (see [15] or [2, page 132]), we may choose a point $a$ in $\Omega-B$ so that these functions are non-vanishing in $z$ near $b \Omega$. By (6.4), we have $\Lambda(a, z) T(z)=-K(a, z) \overline{T(z)}$ and $\Lambda\left(w_{k}, z\right) T(z)=-K\left(w_{k}, z\right) \overline{T(z)}$ for $z \in b \Omega$. Divide the second of these identities by the first and use the fact that $R(z)=z$ on $b \Omega$ to obtain

$$
\frac{\Lambda\left(w_{k}, z\right)}{\Lambda(a, z)}=\frac{K\left(w_{k}, R(z)\right)}{K(a, R(z))} \quad \text { for } z \in b \Omega
$$

The function on the left hand side of (6.1) is holomorphic in $z$ on a neighborhood of $b \Omega$; so is the function on the right hand side. Since these functions agree on $b \Omega$, they must be equal on a neighborhood of $b \Omega$. In fact, because $K$ and $\Lambda$ are rational, these two functions are equal as meromorphic functions on the neighborhood $\mathcal{O}$ of $b \Omega$ on which $R(z)$ is defined. We may assume that $\mathcal{O}$ is small enough that $K(z, a)$ and $\Lambda(z, a)$ have no poles or zeroes in $\mathcal{O}$. Formula (6.5) now allows us to read off the following facts. Neither $K\left(z, w_{k}\right)$ nor $\Lambda\left(z, w_{k}\right)$ can have a pole $z_{0} \in D_{1}$ near $\gamma_{1}$ because neither of these functions has a pole at the reflected point $R\left(z_{0}\right) \in \Omega$.

Notice that (6.4) yields that

$$
\Lambda\left(z, A_{n}\right) T\left(A_{n}\right)=-K\left(z, A_{n}\right) \overline{T\left(A_{n}\right)}
$$

for $z \in \Omega$, and hence for $z$ in $D_{1}$. Hence, $K\left(z, A_{n}\right)$ and $\Lambda\left(z, A_{n}\right)$ have the same poles in $\overline{D_{1}}$. Because no poles of $K\left(z, w_{k}\right)$ or $\Lambda\left(z, w_{k}\right)$ can migrate near the boundary of $D_{1}$ as $w_{k} \rightarrow A_{n}$, we deduce that $P_{K}=P_{\Lambda}$.

We now take a sequence of points $w_{k}$ in $\Omega$ that tend to $A_{1}$ along $\sigma$. Formula (6.5) remains valid and, if we reason as above, we deduce that, since $\Lambda\left(z, w_{k}\right)$ has a double pole at $z=w_{k}$, it follows that $K\left(z, w_{k}\right)$ has a double pole at the reflected point $R\left(w_{k}\right)$. We now let $k \rightarrow \infty$ and use the facts that $K\left(z, A_{1}\right)$ and $\Lambda\left(z, A_{1}\right)$ have the same poles in $\overline{D_{1}}$ and that one of those poles is a double pole at $A_{1}$. We deduce that $P_{K}=P_{\Lambda}+2$. This relationship contradicts the one we obtained by letting $w_{k}$ tend to $A_{n}$, and we conclude that $\Omega$ cannot be multiply connected. The proof is complete. 
We shall now explain how to relax the smoothness assumption that the boundary of $\Omega$ be $C^{\infty}$ smooth in Theorem 6.1. The conclusion about the Szego" kernel holds if the boundary is only assumed to be $C^{2}$ smooth because, in this setting, the functions $S_{a}(z)$ and $L_{a}(z)$ extend continuously to the boundary and $T(z)$ is continuous on $b \Omega$. All of the arguments carry through as before.

We next show that the conclusion about the Bergman kernel in Theorem 6.1 holds if the domain $\Omega$ is only assumed to be finitely connected and such that no boundary component is a point. Since the Bergman kernel is related to the Green's function by $K(z, w)=(-2 / \pi)\left(\partial^{2} / \partial z \partial \bar{w}\right) G(z, w)$, it follows that if the Green's function is $\frac{1}{2}$ the logarithm of a rational function of the real and imaginary parts of $z$ and $w$, then the Bergman kernel must be rational, too. The Green's functions associated to the unit disc is given by

$$
G(z, w)=-\frac{1}{2} \ln \left|\frac{z-w}{1-z \bar{w}}\right|^{2}
$$

and the transformation formula for the Green's functions under biholomorphic mappings can be used to see that the Green's function associated to a simply connected domain is $\frac{1}{2}$ the logarithm of a rational function of the real and imaginary parts of $z$ and $w$ if and only if the there is a rational biholomorphic mapping of the domain onto the unit disc. Hence, we will obtain a proof of the following theorem.

Theorem 6.3. Suppose $\Omega$ is a finitely connected domain such that no boundary component of $\Omega$ is a point. The Green's function $G(z, a)$ associated to $\Omega$ is $\frac{1}{2}$ the logarithm of a real valued rational function of the four real variables given by the real and imaginary parts of $z$ and $a$ if and only if $\Omega$ is simply connected and there is a rational biholomorphic mapping of $\Omega$ onto the unit disc. Similarly, the Bergman kernel $K(z, w)$ associated to $\Omega$ is rational if and only if $\Omega$ is simply connected and there is a rational biholomorphic mapping of $\Omega$ onto the unit disc.

Hence, the only finitely connected domains having Green's functions as simple as the Green's function for the disc are the obvious ones. (Of course, the Green's function itself can never be rational because it has a logarithmic singularity.)

Proof of Theorem 6.3. Suppose $\Omega$ is an $n$-connected domain such that no boundary component is a point and assume that the Bergman kernel $K(z, w)$ associated to $\Omega$ is rational. It is a standard result in the theory of conformal mapping that $\Omega$ is biholomorphic to a bounded domain with real analytic boundary. Let $\tilde{\Omega}$ denote such a bounded $n$-connected domain with $C^{\infty}$ smooth boundary whose boundary consists of $n$ non-intersecting simple closed real analytic curves 
and let $\Phi: \Omega \rightarrow \tilde{\Omega}$ denote the biholomorphic mapping. Let $\tilde{K}(z, w)$ denote the Bergman kernel associated to $\tilde{\Omega}$. The transformation formula for the Bergman kernel under biholomorphic mappings gives

$$
K(z, w)=\Phi^{\prime}(z) \tilde{K}(\Phi(z), \Phi(w)) \overline{\Phi^{\prime}(w)} .
$$

It will be convenient to operate in the extended complex plane because it is inconvenient if the point at infinity belongs to one of the boundary components of $\Omega$. The transformation formula for the Bergman kernel under biholomorphic maps allows us to replace $\Omega$ by any domain which is the inverse image of $\Omega$ under a rational biholomorphic map. By replacing $\Omega$ by its inverse image under a mapping of the form $1 /(z-a)$, we may suppose that $\Omega$ contains the point at infinity in its interior.

Since the transformation formula for the Bergman kernels under proper holomorphic mappings holds in the more general setting of Theorem 6.3, we deduce, as above, that the Ahlfors mappings are rational when the Bergman kernel is rational. Pick a point $a \in \Omega$ and let $f_{a}(z)$ denote the Ahlfors map associated to $a$. Since $f_{a}$ is rational, and since it is clear that $f_{a}$ cannot have any poles in $\bar{\Omega}$, it follows that the boundary of $\Omega$ consists of finitely many piecewise real analytic curves. Furthermore, there are at most finitely many points in the boundary where the boundary is not a $C^{\infty}$ smooth curve. The non-smooth points in the boundary occur at boundary points where $f_{a}^{\prime}$ vanishes. Suppose $f_{a}^{\prime}$ vanishes to order $m$ at a boundary point $z_{0}$. The boundary of $\Omega$ near $z_{0}$ is described by two real analytic curves that cross at $z_{0}$ and make an angle of $\pi /(m+1)$. The mapping $\Phi: \Omega \rightarrow \tilde{\Omega}$ described above extends continuously to the boundary of $\Omega$. Let $A=\Phi(a)$, and let $F_{A}(z)$ denote the Ahlfors map of $\tilde{\Omega}$ onto the unit disc associated to $A$. Since Ahlfors maps are solutions to an extremal problem of mapping the domain into the unit disc in such a way so as to maximize the real part of the derivative of the mapping at the associated point, it is easy to see that Ahlfors maps are invariant under biholomorphic mappings modulo unimodular constants to make derivatives real valued at the points of interest. Hence, we may write

$$
f_{a}=e^{i \vartheta} F_{A} \circ \Phi
$$

where $\vartheta$ is a real constant. Since $\tilde{\Omega}$ has real analytic boundary, the Ahlfors map $F_{A}$ extends holomorphically past the boundary and is locally one-to-one near the boundary. Hence, near $z_{0}$, we may write

$$
\Phi=F_{A}^{-1} \circ\left(e^{-i \vartheta} f_{a}\right)
$$

to see that $\Phi$ extends holomorphically past the boundary of $\Omega$ near $z_{0}$ and $\Phi^{\prime}$ vanishes to order $m$ at $z_{0}$. Hence $\Phi$ extends holomorphically to a neighborhood of 
$\bar{\Omega}$ and $\Phi^{\prime}$ only vanishes at points in $\bar{\Omega}$ that are corners in the boundary. Formula (6.6) now yields that $K(z, w)$ extends holomorphically in $z$ and antiholomorphically in $w$ to a neighborhood in $\mathbb{C} \times \mathbb{C}$ of $(\bar{\Omega} \times \bar{\Omega})-\{(z, z): z \in b \Omega\}$. The rest of the argument is now a routine transcription of the proof of Theorem 6.1. All of the kernel identities used in the proof of Theorem 6.1 can be deduced by pulling back the identities that are known on $\tilde{\Omega}$. For example, the fact that $\left|f_{a}(z)\right|=\left|f_{z}(a)\right|$ can be deduced by using the argument given in the proof of Theorem 6.1 and then pulling back to $\Omega$ using $\Phi$. The Green's function $G(z, w)$ is related to the Green's function $\tilde{G}(z, w)$ on $\tilde{\Omega}$ via $G(z, w)=G(\Phi(z), \Phi(w))$ and the corresponding statement for the $\Lambda$ kernels is $\Lambda(x, w)=\Phi^{\prime}(z) \tilde{\Lambda}(\Phi(z), \Phi(w)) \Phi^{\prime}(w)$. The movement of zeroes and poles of $K(z, w)$ near the boundary of $\Omega$ can be read off from (6.6) and the known behavior of the zeroes and poles of $\tilde{K}(z, w)$ near the boundary of $\tilde{\Omega}$. The kernel $K(z, w)$ vanishes identically when $z=z_{0}$ is a corner in the boundary, but this does not interfere with our work because we may choose a curve $\sigma$, as in the proof of Theorem 6.1, that does not begin or terminate at a corner in the boundary of $\Omega$. As $w$ moves along such a curve, the poles of $K(z, w)$ as a function of $z$ that lie in a bounded component $\overline{D_{1}}$ of the complement of $\Omega$ cannot approach a corner in $b \Omega$. We leave it to the reader to complete the proof.

7. Complexity of the Poisson kernel. I showed in [4] how the Szegö projection can be used to solve the Dirichlet problem. The method gives rise to a formula for the Poisson kernel of a bounded $n$-connected domain $\Omega$ with $C^{\infty}$ smooth boundary which, in light of results in Section 4, reveals the level of complexity of that kernel. We shall use the same notation for describing $\Omega$ as we have set up previously, and as before, we also select a point $a \in \Omega$ such that the zeroes $a_{1}, \ldots, a_{n-1}$ of $S(z, a)$ are all distinct and simple. As before, let $S_{a}(z)=S(z, a)$ and $L_{a}(z)=L(z, a)$. The Szegö projection $P$ associated to $\Omega$ is the orthogonal projection of $L^{2}(b \Omega)$ onto the Hardy space $H^{2}(b \Omega)$. The Szegö kernel is the kernel for the Szegö projection in the sense that, given a function $u \in L^{2}(b \Omega)$, the projection $P u$ is identified with a holomorphic function $h=P u$ defined on $\Omega$ whose $L^{2}$ boundary values are equal to $P u$, and

$$
(P u)(z)=\int_{w \in b \Omega} S(z, w) u(w) d s
$$

The Szegö projection maps $C^{\infty}(b \Omega)$ into $C^{\infty}(\bar{\Omega})$ (see [2] for proofs of these basic facts).

Recall that the set of functions $\left\{L\left(z, a_{k}\right) S(z, a)\right\}_{k=1}^{n-1}$ spans the same linear space as the set of functions $\left\{F_{k}^{\prime}\right\}_{k=1}^{n-1}$. Define an $(n-1) \times(n-1)$ matrix of 
periods via

$$
A_{j k}=-i \int_{\gamma_{j}} L\left(z, a_{k}\right) S(z, a) d z
$$

for $j=1, \ldots, n-1$. Because the matrix of periods of $F_{k}^{\prime}$ is non-singular, so is $\left[A_{j k}\right]$. The following theorem was proved in [4].

Theorem 7.1. Given $\varphi \in C^{\infty}(b \Omega)$, let $c_{j}$ solve the linear system

$$
\sum_{j=1}^{n-1} A_{j k} c_{j}=P\left(S_{a} \varphi\right)\left(a_{k}\right), \quad k=1, \ldots, n-1
$$

The harmonic extension $\mathcal{E} \varphi$ of $\varphi$ to $\Omega$ is given by

$$
\mathcal{E} \varphi=h+\bar{H}+\sum_{j=1}^{n-1} c_{j} \omega_{j}
$$

where, if we let $\psi=\varphi-\sum_{j=1}^{n-1} c_{j} \omega_{j}$, then

$$
h=\frac{P\left(S_{a} \psi\right)}{S_{a}}
$$

and

$$
H=\frac{P\left(L_{a} \bar{\psi}\right)}{L_{a}} .
$$

The functions $h$ and $H$ are in $A^{\infty}(\Omega)$.

This theorem allows the Poisson kernel to be written down in terms of the Szegö and Garabedian kernels. Let $\left[B_{j k}\right]$ denote the inverse of $\left[A_{j k}\right]$ so that $c_{j}=\sum_{k=1}^{n-1} B_{j k} P\left(S_{a} \varphi\right)\left(a_{k}\right)$, i.e., so that

$$
c_{j}=\int_{w \in b \Omega}\left(\sum_{k=1}^{n-1} B_{j k} S\left(a_{k}, w\right) S(w, a)\right) \varphi(w) d s .
$$

The formulas for $h$ and $H$ can be written

$$
h(z)=\int_{w \in b \Omega} \frac{S(z, w) S(w, a)}{S(z, a)} \psi(w) d s
$$


and

$$
H(z)=\int_{w \in b \Omega} \frac{S(z, w) L(w, a)}{L(z, a)} \overline{\psi(w)} d s .
$$

Finally, when all these formulas are collected in one sum, we see that the Poisson extension $\mathcal{E} u$ of $u$ to $\Omega$ is given by an integral

$$
(\mathcal{E} u)(z)=\int_{w \in b \Omega} p(z, w) u(w) d s,
$$

where $p(z, w)$ is the Poisson kernel and is given by

$$
\begin{aligned}
p(z, w)=\frac{S(z, w) S(w, a)}{S(z, a)}+\frac{\overline{S(z, w) L(w, a)}}{\overline{L(z, a)}} & \\
& -\sum_{j, k=1}^{n-1}\left(B_{j k} S\left(a_{k}, w\right) S(w, a) \int_{\zeta \in \gamma_{j}} \frac{S(z, \zeta) S(\zeta, a)}{S(z, a)} d s\right) \\
& -\sum_{j, k=1}^{n-1}\left(\frac{\overline{S(z, \zeta) L(\zeta, a)}}{\overline{B_{j k} S\left(a_{k}, w\right) S(w, a)}} \int_{\zeta \in \gamma_{j}} \frac{\overline{L(z, a)}}{n-1}\right) \\
+ & \sum_{j=1}^{n} \omega_{j}(z)\left(\sum_{k=1}^{n-1} B_{j k} S\left(a_{k}, w\right) S(w, a)\right) .
\end{aligned}
$$

The first term in the sum on the right hand side of (7.2) is a meromorphic function in $z$ with simple poles at the zeroes of $S(z, a)$. The second term is antiholomorphic in $z$ with no poles. The third term is meromorphic in $z$ with simple poles at the zeroes of $S(z, a)$ that exactly cancel the simple poles of the first term. The fourth term is antiholomorphic in $z$ with no poles. The last term is harmonic in $z$.

Formula (7.2) is in a rather raw state. We shall now show how this formula can be refined. Along the way, we shall see that there is a formula relating the Poisson kernel to the Poisson-Szegö kernel in multiply connected domains.

We may rewrite $(7.2)$ in the form

$$
p(z, w)=h(z, w)+\overline{H(z, w)}+\sum_{j=1}^{n-1} \omega_{j}(z) \mu_{j}(w)
$$

where $h(z, w)$ and $H(z, w)$ are functions in $C^{\infty}(\bar{\Omega} \times b \Omega-\{(w, w): w \in b \Omega\})$ which are holomorphic in $z$ for fixed $w \in b \Omega$ and

$$
\mu_{j}(w)=\sum_{k=1}^{n-1} B_{j k} S\left(a_{k}, w\right) S(w, a)
$$


is a $C^{\infty}$ function on $b \Omega$. Since the Poisson kernel is a real valued function, the sum on the right hand side of (7.3) is real valued. We now claim that $\mu_{j}(w)$ must be real valued on $b \Omega$. Indeed, if it were not, by taking the imaginary part of (7.3) at a point $w \in b \Omega$ where $\mu_{j}(w) \neq 0$, we could express a non-trivial linear combination of the functions $\left\{\omega_{j}(z): j=1, \ldots, n-1\right\}$ as the imaginary part of a holomorphic function. Since this is impossible, we conclude that each $\mu_{j}(w)$ must be real valued. A similar argument shows that $\mu_{j}(w)$ does not depend on $a$. Indeed, if $\mu_{j}(w, a)$ and $\mu_{j}(w, \tilde{a})$ are the functions obtained using $a$ and $\tilde{a}$, respectively, and if $\left(\mu_{j}(w, a)-\mu_{j}(w, \tilde{a})\right) \neq 0$ at a point $w \in b \Omega$, we could subtract the corresponding formulas for $p(z, w)$ in (7.2) and thereby express a non-trivial linear combination of the functions $\left\{\omega_{j}(z): j=1, \ldots, n-1\right\}$ as the real part of a holomorphic function. This is impossible, and so $\mu_{j}(w)$ does not depend on the choice of $a$. Later, we shall need to know that the functions $\mu_{j}$ are linearly independent. Integrate (7.3) against $\omega_{k}(w) d s$ to obtain an identity,

$$
\omega_{k}(z)=h_{k}(z)+\overline{H_{k}(z)}+\sum_{j=1}^{n-1} c_{k j} \omega_{j}(z)
$$

where $c_{k j}=\int_{\gamma_{k}} \mu_{j} d s$ and $h_{k}$ and $H_{k}$ are holomorphic. Since no non-trivial linear combination of the functions $\omega_{j}(z)$ can be equal to the real part of a holomorphic function, we conclude that $c_{k j}=1$ if $k=j$ and $c_{k j}=0$ if $k \neq j$. This shows that the $\mu_{j}$ are independent.

We next investigate the dependence of $h(z, w)$ and $H(z, w)$ on $a$. We shall see that $h$ and $H$ are determined uniquely by the condition that $H(a, w)=0$ for each $w \in b \Omega$. Since $p(z, w), \mu_{j}(w)$ and $\omega_{j}(z)$ are real valued, it follows that, for each $w \in b \Omega, h(z, w)$ and $H(z, w)$ are holomorphic functions of $z$ with the same imaginary part. Hence, they differ by a constant. By setting $z=a$, we may evaluate that constant. Since $L(z, a)$ has a pole at $z=a$, we see that $H(a, w)=0$, and so

$$
\begin{aligned}
h(z, w)-H(z, w) & =h(a, w)-H(a, w)=h(a, w) \\
& =\frac{|S(w, a)|^{2}}{S(a, a)}-\sum_{j=1}^{n-1} \mu_{j}(w) \int_{\zeta \in \gamma_{j}} \frac{|S(\zeta, a)|^{2}}{S(a, a)} d s .
\end{aligned}
$$

Let

$$
\lambda_{j}(a)=\int_{\zeta \in \gamma_{j}} \frac{|S(\zeta, a)|^{2}}{S(a, a)} d s
$$


Since $h(z, w)=H(z, w)+h(a, w)$ where $h(a, w)$ is real, we obtain that $h(z, w)+$ $\overline{H(z, w)}=(2 \operatorname{Re} H(z, w))+h(a, w)$, and so

$$
\begin{gathered}
p(z, w)=2 \operatorname{Re}\left[\frac{S(z, w) L(w, a)}{L(z, a)}-\sum_{j=1}^{n-1} \mu_{j}(w) \int_{\zeta \in \gamma_{j}} \frac{S(z, \zeta) L(\zeta, a)}{L(z, a)} d s\right] \\
+\frac{|S(w, a)|^{2}}{S(a, a)}+\sum_{j=1}^{n-1}\left(\omega_{j}(z)-\lambda_{j}(a)\right) \mu_{j}(w) .
\end{gathered}
$$

Identity (7.4) has the virtue that none of the holomorphic functions appearing in denominators have zeroes. I think that formula (7.4), together with (3.2), offer an excellent strategy for computing the Poisson kernel efficiently.

Finally, we may set $z=a$ in (7.4) to obtain a formula that will fit on one line,

$$
p(a, w)=\frac{|S(w, a)|^{2}}{S(a, a)}+\sum_{j=1}^{n-1}\left(\omega_{j}(a)-\lambda_{j}(a)\right) \mu_{j}(w) .
$$

This last formula relates the Poisson kernel $p(z, w)$ to the Poisson-Szegö kernel $|S(w, a)|^{2} / S(a, a)$ in a multiply connected domain. (These two kernels are equal in simply connected domains.) We have shown that (7.5) is valid when $a \in \Omega$ is a point where the $n-1$ zeroes of $S(z, a)$ as a function of $z$ are all simple zeroes. However, it is clear that (7.5) is valid for all $a \in \Omega$ because the functions in it are all continuous, $S(a, a)>0$, and the set of points $a$ where the zeroes of $S(z, a)$ are not simple is finite.

The functions $\lambda_{j}(a)$ are rather interesting. We shall now prove that $\lambda_{j}(a)$ is a non-harmonic function of $a$ in $C^{\infty}(\bar{\Omega})$. Because $S(a, a) \rightarrow \infty$ as $a \rightarrow b \Omega$, and because $\int_{b \Omega}|S(z, a)|^{2} d s=S(a, a)$, it is easy to see that $\lambda_{j}(a) \rightarrow 1$ as $a \rightarrow \gamma_{j}$ and $\lambda_{j}(a) \rightarrow 0$ as $a \rightarrow \gamma_{k}, k \neq j$. Let $w_{1}, w_{2}, \ldots, w_{n-1}$ be $n-1$ distinct points in $b \Omega$ and consider the determinant $\operatorname{det}\left[\mu_{j}\left(w_{k}\right)\right]$. We claim that it is possible to choose $w_{1}, \ldots, w_{n-1}$ so that this determinant is non-zero. To see this, suppose the determinant is zero for any choice of the points $w_{k}$. Replace $w_{n-1}$ by a variable $w$ and expand the determinant along the bottom row to obtain a linear combination of the functions $\mu_{j}(w)$ that sums to the zero function. The coefficients in this linear combination must be zero because the $\mu_{j}$ are independent. Hence, the determinants of all the principle minors of the matrix must also vanish. Since none of the $\mu_{j}$ are identically zero, this argument can be repeated for smaller and smaller submatrices of the original matrix until a contradiction is obtained. We may now suppose that the $w_{k}$ have been chosen so that $0 \neq \operatorname{det}\left[\mu_{j}\left(w_{k}\right)\right]$. Apply the Laplace operator to (7.5) in the $a$ variable and replace $w$ by $w_{k}$. The functions 
$\omega_{j}(a)$ and $p(a, w)$ are harmonic in $a$. We may now deduce that $\Delta \lambda_{j}(a)$ is equal to the Laplace operator applied to a linear combination of $\left|S\left(w_{k}, a\right)\right|^{2} / S(a, a)$. Hence, $\Delta \lambda_{j}(a)$ is in $C^{\infty}\left(\bar{\Omega}-\left\{w_{1}, \ldots, w_{n-1}\right\}\right)$. We may now move the points $w_{k}$ slightly, keeping the determinant non-zero, to see that, in fact, $\Delta \lambda_{j}(a)$ is in $C^{\infty}(\bar{\Omega})$. Since $\lambda_{j}$ has continuous boundary values of one on $\gamma_{j}$ and zero on $\gamma_{k}$ for $k \neq j$, elliptic regularity yields that $\lambda_{j}$ is in $C^{\infty}(\bar{\Omega})$. The function $u(a)=|S(w, a)|^{2} / S(a, a)$ cannot be harmonic in $a$ on $\Omega$ for fixed $w \in b \Omega$. Indeed, if it were harmonic in $a$, it would be a positive harmonic function in $C^{\infty}(\bar{\Omega}-\{w\})$ that vanishes on $b \Omega-\{w\}$. Recall that $S(a, a)^{-1}$ was shown to be a function in $C^{\infty}(\bar{\Omega})$ that vanishes on $b \Omega$ and that has non-vanishing normal derivative at each boundary point of $\Omega$. The Hopf Lemma would imply that the normal derivative of $u(a)$ must be non-zero at all boundary points in $b \Omega-\{w\}$. But $S(w, a)$ vanishes at $n-1$ distinct boundary points $a=w_{1}, w_{2}, \ldots, w_{n-1}$ (see [4]). At such points, the normal derivative of $u(a)$ is zero. This contradiction lets us conclude that $u(a)$ cannot be harmonic. It follows that the functions $\lambda_{j}$, $j=1, \ldots, n-1$ cannot be harmonic.

The Poisson-Szegő kernel $|S(w, a)|^{2} / S(a, a)$ is equal to the Poisson kernel in a simply connected domain, and so it is harmonic in $a$ on a simply connected domain. We remark here that we proved in the last paragraph that the Laplace operator in the $a$ variable applied to the Poisson-Szegö kernel is given by

$$
\Delta_{a}\left(\frac{|S(w, a)|^{2}}{S(a, a)}\right)=\sum_{j=1}^{n-1} \mu_{j}(w) \Delta_{a} \lambda_{j}(a)
$$

which, although it is not zero, it is in $C^{\infty}(b \Omega \times \bar{\Omega})$ as a function of $(w, a) \in$ $b \Omega \times \bar{\Omega}$.

We shall now examine formula (7.4) in more detail to shed light on the complexity of the Poisson kernel. We can use (2.1) to replace $L(w, a)$ by $i S(a, w) \overline{T(w)}$ in the formula. Furthermore, we may use (2.1) again to write

$$
\mu_{j}(w)=\sum_{k=1}^{n-1} B_{j k} S\left(a_{k}, w\right) S(w, a)=\sum_{k=1}^{n-1} i B_{j k} S\left(a_{k}, w\right) \overline{L(w, a) T(w)} .
$$

It follows that $\mu_{j}(w)=\overline{g_{j}(w) T(w)}$ where $g_{j} \in A^{\infty}(\Omega)$. (Although we shall not need this fact, it also follows that

$$
\mu_{j}(w)=\sum_{k=1}^{n-1} c_{k} F_{k}^{\prime}(w) T(w)
$$

because the linear span of the functions $S\left(w, a_{k}\right) L(w, a)$ is the same as the linear span of the functions $F_{j}^{\prime}(w)$, and $F_{j}^{\prime}(w) T(w)=-\overline{F_{j}^{\prime}(w) T(w)}$ on $b \Omega$.) Any $C^{\infty}$ 
function on the boundary of $\Omega$ can be written as a sum $h(w)+\overline{H(w) T(w)}$ where $h$ and $H$ are in $A^{\infty}(\Omega)$. We can find an explicit decomposition of this form for the Poisson-Szegö kernel by using (2.1) as follows:

$$
\begin{aligned}
\frac{|S(w, a)|^{2}}{S(a, a)} & =\frac{S(w, a) S(a, w)}{S(a, a)} \\
& =\frac{[S(w, a)-S(a, a)] L(w, a) T(w)}{i S(a, a)}+\frac{1}{i} L(w, a) T(w) \\
& =\frac{[S(w, a)-S(a, a)] L(w, a) T(w)}{i S(a, a)}+S(a, w) \\
& =\overline{h_{0}(w)}+H_{0}(w) T(w)
\end{aligned}
$$

where $h_{0}$ and $H_{0}$ are in $A^{\infty}(\Omega)$ as functions of $w$. Note that $\overline{h_{0}(w)}+H_{0}(w) T(w)=$ $h_{0}(w)+\overline{H_{0}(w) T(w)}$ because the sum is real valued. Finally, we may write the Poisson kernel as

$$
p(z, w)=\operatorname{Re}\left[\begin{array}{l}
\frac{\sum_{i, j=1}^{n} h_{i}(z) \overline{H_{j}(w) T(w)}}{1-f(z) \overline{f(w)}}+\sum_{j=1}^{n-1} A_{j}(z) \overline{B_{j}(w) T(w)} \\
+h_{0}(w)+\overline{H_{0}(w) T(w)}+\sum_{j=1}^{n-1} \omega_{j}(z) \overline{g_{j}(w) T(w)}
\end{array}\right.
$$

where $f(z)$ denotes an Ahlfors mapping of $\Omega$ onto the unit disc, and the other functions, $h_{j}, H_{j}, A_{j}, B_{j}$, and $g_{j}$, are all holomorphic functions of one variable in $A^{\infty}(\Omega)$. Recall that Ahlfors maps are also in $A^{\infty}(\Omega)$. Hence, the Poisson kernel is formed by taking simple combinations of the functions $\omega_{j}$ and finitely many holomorphic functions in $A^{\infty}(\Omega)$ of one variable.

We now to turn to the question of whether or not the Poisson kernel can be a rational function of the variable $z \in \Omega$.

Theorem 7.2. Suppose that $\Omega$ is a bounded $n$-connected domain with $C^{\infty}$ smooth boundary. The Poisson kernel $p(z, w)$ associated to $\Omega$ is such that $p(z, w)$ is a rational function of the real and imaginary parts of $z \in \Omega$ for $w$ in an open subset of the boundary if and only if $n=1$ and $\Omega$ is biholomorphic to the unit disc via a rational map $f: \Omega \rightarrow D_{1}(0)$.

It is easy to reduce the smoothness hypothesis on $b \Omega$ in Theorem 7.2 from $C^{\infty}$ to $C^{2}$ smooth. I leave it to others to study just how far the smoothness can be reduced. 
Proof of Theorem 7.2. Let $G(z, w)$ denote the classical Green's function associated to $\Omega$. It is well known that the Poisson kernel is related to the normal derivative of the Green's function,

$$
p(z, w)=\frac{1}{2 \pi} \frac{\partial}{\partial n_{w}} G(z, w), \quad z \in \Omega, w \in b \Omega,
$$

where $\left(\partial / \partial n_{w}\right)$ denotes the normal derivative in the $w$ variable. Since the Green's function vanishes as a function of $w$ on $b \Omega$ when $z \in \Omega$, the tangential derivative of the Green's function in the $w$ variable is zero. Let $\zeta(s)$ denote a parametrization of $b \Omega$ with respect to arc length $s$. We may write

$$
0=\frac{d}{d s} G(z, \zeta(s))=\frac{\partial G}{\partial \zeta}(z, \zeta(s)) \zeta^{\prime}(s)+\frac{\partial G}{\partial \bar{\zeta}}(z, \zeta(s)) \overline{\zeta^{\prime}(s)},
$$

and, from this, we can deduce that

$$
\frac{\partial G}{\partial w}(z, w) T(w)=-\frac{\partial G}{\partial \bar{w}}(z, w) \overline{T(w)} \quad \text { for } w \in b \Omega, z \in \Omega .
$$

If $\varphi$ is harmonic on $\Omega$ near a point $w_{0} \in b \Omega$ and $\varphi$ is $C^{\infty}$ smooth up to the boundary near $w_{0}$, then $\varphi$ can be expressed as a sum $\varphi(w)=h(w)+\overline{H(w)}$ where $h$ and $H$ are holomorphic functions on $\Omega \cap D_{\varepsilon}\left(w_{0}\right), \varepsilon>0$, that extend $C^{\infty}$ smooth up to the boundary near $w_{0}$. The Cauchy-Riemann equations can be used to see that the normal derivative of $\varphi$ near $w_{0}$ is given by

$$
\frac{\partial \varphi}{\partial n_{w}}(w)=-i h^{\prime}(w) T(w)+i \overline{H^{\prime}(w) T(w)}, \quad w \in b \Omega .
$$

Near such a point $w_{0}$, we may express $G(z, w)$ as $G(z, w)=h(w)+\overline{h(w)}$, and we may use (7.6) together with the simple fact that $h^{\prime}(w)=2(\partial / \partial w) \operatorname{Re} h(w)=$ $2(\partial / \partial w) \frac{1}{2} G(z, w)$ to deduce that

$$
\begin{aligned}
\frac{\partial G}{\partial n_{w}}(z, w) & =-i \frac{\partial G}{\partial w}(z, w) T(w)+i \frac{\partial G}{\partial \bar{w}}(z, w) \overline{T(w)} \\
& =2 i \frac{\partial G}{\partial \bar{w}}(z, w) \overline{T(w)}
\end{aligned}
$$$$
w \in b \Omega \text {. }
$$

It follows that

$$
\frac{\partial p}{\partial z}(z, w)=\frac{i}{\pi} \frac{\partial^{2} G}{\partial z \partial \bar{w}}(z, w) \overline{T(w)}
$$

for $w \in b \Omega$ and $z \in \Omega$. Because the Bergman kernel $K(z, w)$ associated to $\Omega$ is equal to $(-2 / \pi)\left(\partial^{2} / \partial z \partial \bar{w}\right) G(z, w)$, it follows that, if $p(z, w)$ is a rational function of the real and imaginary parts of $z \in \Omega$ for $w$ in an open subset of the boundary, 
then $K(z, w)$ is a rational function of $z$ for $z \in \Omega$ for $w$ in an open subset of the boundary. The proof will be finished (via Theorems 6.1 and 6.2) if we show that such a condition implies that the Bergman kernel is rational.

Suppose that $K(z, w)$ is a rational function of $z$ for $w$ in an open subset $\mathcal{O} \subset b \Omega$. This means that for each $w \in \mathcal{O}$, there are positive integers $M(w)$ and $N(w)$, and coefficients $A_{k}(w)$ and $B_{k}(w)$ such that

$$
K(z, w)\left(\sum_{k=0}^{N(w)} A_{k}(w) z^{k}\right)+\sum_{k=0}^{M(w)} B_{k}(w) z^{k}=0 .
$$

By insisting that the polynomials in (7.7) have no common factors, we uniquely specify $M(w)$ and $N(w)$. Since $K(z, w)$ cannot be identically zero in the $z$ variable for any $w \in b \Omega$, we may normalize the coefficients so that

$$
\sum_{k=1}^{N(w)}\left|A_{k}(w)\right|^{2}+\sum_{k=1}^{M(w)}\left|B_{k}(w)\right|^{2}=1 .
$$

It is now a simple matter to show that the set $\mathcal{O}_{N, M}$ of points in $w \in \mathcal{O}$ such that $M(w) \leq M$ and $N(w) \leq N$ is closed in $\mathcal{O}$, and since $\mathcal{O}=\cup \mathcal{O}_{N, M}$, an application of the Baire category theorem yields that there is a non-empty open subset of $b \Omega$ on which $M(w)$ and $N(w)$ are uniformly bounded. Now let $\mathcal{O}$ denote this (possibly smaller) open set. By allowing some coefficients to be zero, we may assume that equations (7.7) and (7.8) hold with $N$ and $M$ in place of $N(w)$ and $M(w)$ for all $w \in \mathcal{O}$. Let $q=N+M$. If we write (7.7) for $q$ points $z_{1}, z_{2}, \ldots, z_{q}$ in $\Omega$, we obtain a linear system, and (7.8) implies that

$$
\operatorname{det}\left[\begin{array}{cccccccc}
K\left(z_{1}, w\right) & K\left(z_{1}, w\right) z_{1} & \cdots & K\left(z_{1}, w\right) z_{1}^{N} & 1 & z_{1} & \cdots & z_{1}^{M} \\
K\left(z_{2}, w\right) & K\left(z_{2}, w\right) z_{2} & \cdots & K\left(z_{2}, w\right) z_{2}^{N} & 1 & z_{2} & \cdots & z_{2}^{M} \\
\vdots & \vdots & \ddots & \vdots & \vdots & \vdots & \ddots & \vdots \\
K\left(z_{q}, w\right) & K\left(z_{q}, w\right) z_{q} & \cdots & K\left(z_{q}, w\right) z_{q}^{N} & 1 & z_{q} & \cdots & z_{q}^{M}
\end{array}\right] \equiv 0
$$

for all $z_{1}, \ldots, z_{q}$ in $\Omega$ and $w \in \mathcal{O}$. Let $\mathbb{K}$ denote the matrix inside this determinant. We may select $z_{1}, \ldots, z_{q-1}$ to be distinct points in $\Omega$. We now replace $z_{q}$ by a variable $z$. If we expand the determinant along the bottom row, we obtain an equation of the form of (7.7) where the coefficients $A_{k}(w)$ and $B_{k}(w)$ are determinants of principal minors of $\mathbb{K}$, and as such, are seen to be the boundary values of antiholomorphic functions of $w$ on $\Omega$ that extend $C^{\infty}$ smoothly to the boundary. Therefore, this identity extends to hold for all $w \in \Omega$. Suppose for now that at least one of the $B_{k}(w)$ is not identically zero on $\mathcal{O}$. It would then follow that $K(z, w)$ is rational in $z$ for $w$ in an open subset of $\Omega$, and this implies that $K(z, w)$ is rational (see the remarks before Theorem 6.1 ). If all the $B_{k}(w)$ 
vanish on $\mathcal{O}$, we must resort to the following argument. Note that, since $K(z, w)$ cannot vanish identically in $z$ when $w \in b \Omega$, there are plenty of $1 \times 1$ submatrices of $\mathbb{K}$ that contain Bergman kernel terms whose determinants are not identically zero. Let $m$ be the largest positive integer such that the determinant of every $m \times m$ submatrix of $\mathbb{K}$ vanishes identically in all the variables, but that there is an $(m-1) \times(m-1)$ submatrix with non-vanishing determinant. Now let $\mathbb{K}$ denote such an $m \times m$ submatrix. It is clear that $\mathbb{K}$ must contain a column with Bergman kernel terms (because any submatrix without Bergman kernel terms is just a Vandermonde-type matrix, and if the $z_{j}$ 's are distinct, it will have non-zero determinant). We can use this new matrix $\mathbb{K}$ as we did above to get an equation of the form of (7.7) in which not all the functions $B_{k}(w)$ vanish identically on $\mathcal{O}$. Such an equation extends to hold for all $w \in \Omega$, and we conclude as above that $K(z, w)$ is rational. The proof is complete.

8. The Green's function. In this section, we show that the gradient of the Green's function associated to a finitely connected domain $\Omega$ with $C^{\infty}$ smooth boundary is composed of finitely many functions of one variable in $C^{\infty}(\bar{\Omega})$. We may use $(2.1)$ to rewrite $(7.5)$ as

$$
p(z, w)=i \frac{S(z, w) \overline{L(w, z) T(w)}}{S(z, z)}+\sum_{j=1}^{n-1}\left(\omega_{j}(z)-\lambda_{j}(z)\right) \overline{g_{j}(w) T(w)}
$$

where, as in Section $7, g_{j}(w)=-i \sum_{k=1}^{n-1} \overline{B_{j k}} S\left(w, a_{k}\right) L(w, a)$. Since,

$$
p(z, w)=\frac{1}{2 \pi} \frac{\partial}{\partial n_{w}} G(z, w)=\frac{i}{\pi} \frac{\partial}{\partial \bar{w}} G(z, w) \overline{T(w)},
$$

we may equate these two expressions to obtain the formula in the following theorem.

Theorem 8.1. Suppose that $\Omega$ is a bounded $n$-connected domain with $C^{\infty}$ smooth boundary. The gradient of the Green's function associated to $\Omega$ can be read off from the formula

$$
\frac{\partial G}{\partial \bar{w}}(z, w)=\pi\left(\frac{S(z, w) \overline{L(w, z)}}{S(z, z)}-i \sum_{j=1}^{n-1}\left(\omega_{j}(z)-\lambda_{j}(z)\right) \overline{g_{j}(w)}\right) .
$$

We have shown that the formula in Theorem 8.1 is valid for $z \in \Omega$ and $w \in b \Omega$. Since the functions on the left and right hand sides of the equation are both antiholomorphic functions of $w$ on $\Omega-\{z\}$ that have the same boundary values, the identity extends to hold for all $z, w \in \Omega, z \neq w$. 
Theorem 3.1 asserts that the Szegö kernel is composed of finitely many functions of one variable. To see that the gradient of the Green's function is also composed of finitely many functions of one variable in $C^{\infty}(\bar{\Omega})$, we need to show that $L(z, w)$ satisfies an identity analogous to (3.2).

Let $z \in \Omega$ and $w \in b \Omega$, and consider formula (3.2). Using identity (2.1) and the fact that $\overline{f_{a}}=1 / f_{a}$ on $b \Omega$, we obtain

$$
L(z, w)=\frac{f_{a}(w)}{f_{a}(z)-f_{a}(w)}\left(c_{0} S(z, a) L(w, a)+\sum_{i, j=1}^{n-1} \bar{c}_{i j} S\left(z, a_{i}\right) L\left(w, a_{j}\right)\right)
$$

Since both sides of this identity are holomorphic in $z$ and $w$, this identity holds for $z, w \in \Omega, z \neq w$. Note that the constants $c_{0}$ and $c_{i j}$ are the same as the constants in (3.2).

We close this paper by remarking that the $\Lambda(z, w)$ kernel can also be expressed in terms of functions of one variable. By combining (6.4), (5.1), and (2.1), we may derive the identity

$$
\Lambda(w, z)=4 \pi L(w, z)^{2}+\sum_{i, j=1}^{n-1} \lambda_{i j} L\left(w, a_{i}\right) S(w, a) S\left(z, a_{j}\right) L(z, a)
$$

where $z \in \Omega$ and $w \in b \Omega$, and the coefficients $\lambda_{i j}$ are the same as those appearing in (5.1). Since both sides of this identity are holomorphic in $z$ and $w$, the identity holds for $z, w \in \Omega, z \neq w$.

\section{REFERENCES}

[1] L. Ahlfors, Complex Analysis, McGraw Hill, New York, 1979.

[2] S. Bell, The Cauchy transform, potential theory, and conformal mapping, CRC Press, Boca Raton, 1992.

[3] - Numerical computation of the Ahlfors map of a multiply connected planar domain, J. Math. Anal. Appl. 120 (1986), 211-217.

[4] The Szegö projection and the classical objects of potential theory in the plane, Duke Math. J. 64 (1991), 1-26.

[5] - Simplicity of the Bergman, Szegö, and Poisson kernel functions, Mathematical Research Letters 2 (1995), 267-277.

[6] Recipes for classical kernel functions associated to a multiply connected domain in the plane, Complex Variables, Theory and Applications (to appear).

[7] S. Bergman, The kernel function and conformal mapping, Math. Surveys 5, Amer. Math. Soc., Providence, 1950.

[8] S. Bochner \& W. Martin, Several Complex Variables, Princeton Univ. Press, Princeton, 1948.

[9] P. Henrici, Applied and computational complex analysis, Vol. 3, John Wiley, New York, 1986. 
[10] M. JEOng, Approximation theorems on mapping properties of the classical kernel functions of complex analysis, Purdue University $\mathrm{PhD}$ Thesis.

[11] - The Szegö kernel and the rational proper mappings between planar domains, Complex Variables Theory and Appl. 23 (1993), 157-162.

[12] N. Kerzman ES E. M. Stein, The Cauchy kernel, the Szegő kernel, and the Riemann mapping function, Math. Ann. 236 (1978), 85-93.

[13] N. Kerzman \& M. Trummer, Numerical conformal mapping via the Szegö kernel, Numerical Conformal Mapping (L. N. Trefethen, ed.), North Holland, Amsterdam, 1986, pp. 111-123.

[14] M. Schiffer, Various types of orthogonalization, Duke Math. J. 17 (1950), 329-366.

[15] N. Suita \& A. Yamada, On the Lu Qi-Keng conjecture, Proc. Amer. Math. Soc. 59 (1976), 222-224.

[16] M. Trummer, An efficient implementation of a conformal mapping method based on the Szego" kernel, SIAM J. Numer. Anal. 23 (1986), 853-872.

This research was supported in part by NSF grant DMS-9302513.

Department of Mathematics

Purdue University

West Lafayette, Indiana 47907

bell@math.purdue.edu

Received: April 7th, 1995. 